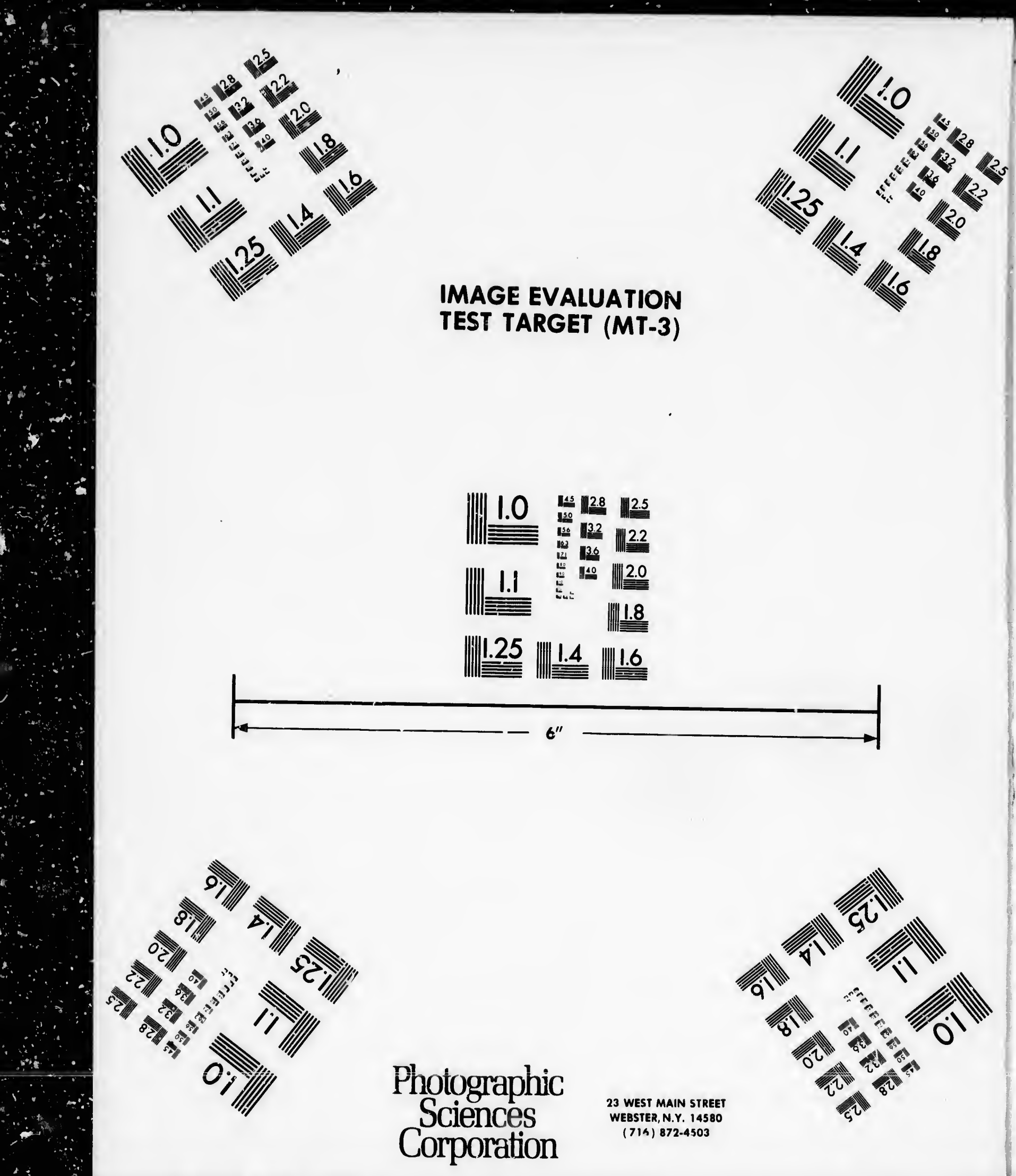


The Institute has attempted to obtain the best original copy available for filming. Features of this copy which may be bibliographically unique. wrich may alter any of the images in the reproduction, or which may significantly change the usual methed of filming, are checked below.

Coloured covers/

Couverture de couleur

Covers damaged/

Couverture endommagé

Covers restored and/or laminated/

Couvertura restaurée et/ou pelliculé

Cover title missing/

Le titre de couverture manque

Colourea maps/

Cartes góographiques en couleur

Coloured ink (i.e. other than blue or black)/

Encre de couleur (i.e. autre que bleue ou noire)

Coloured plates and/or illustrations/

Planches ot/ou iliustrations en couleur

Bound with other material/

Relié avec d'autres documents

Tight binding may cause shadows or distortion along interior margin,

Lare liure serrée peut causer de l'ombre ou de la distorsion le long de la marge intérieure

Blank leaves added curing restoration may appear within the text. Whenever possible, these have been omitted from filming/

II se peut que certaines pages blanches ajoutbes :ors d'une restauration apparaissent dans le texte. mais, lorsque cela était possible, ces pages n'ont pas eté filmées.

Additional comments:/

Commentaires supplémentaires:
L'Institut a microfilmé ie meilleur exemplaire qu'il lui a été possible de se procurer. Les détails de cet exemplaire qui sont peut-etre uniques du point de vue bibliographique, qui peuvent modifier une image reproduite, ou qui peuvent exiger une modification dans la methode normale de filmage sont indiqués ci-dessous.

Coloured pagas/

Pages de couleur

Pages damaged/

Pages endommagées

Pages restored and/or laminated/

Pages restaurées et/ou pelliculees

Pages discoloured, stained or foxed/

Pages décolorées, tachetées ou piquèes

Pages detached/

Pages détachées

Showthrough/

Transparence

Quality of print varies/

Qualité inégale de l'impression

Includes supolementary material/

Comprend du matériel supplémentaire

Only edition available/

Seule édition disponible

Pages wholly or partially obscured by eirata slips, tissues, etc., have been refilmed to ensure the best possible image/

Les pages totalement ou partiellement obscurcies par un feuillet d'errata, une pelure. etc.. cont été filmées à nouveau de façon à obtenir la meilleure image possible.

This item is filmed at the reduction ratio checked below/ Ce document est filmé au taux de réduction indiqué ci-dessous.

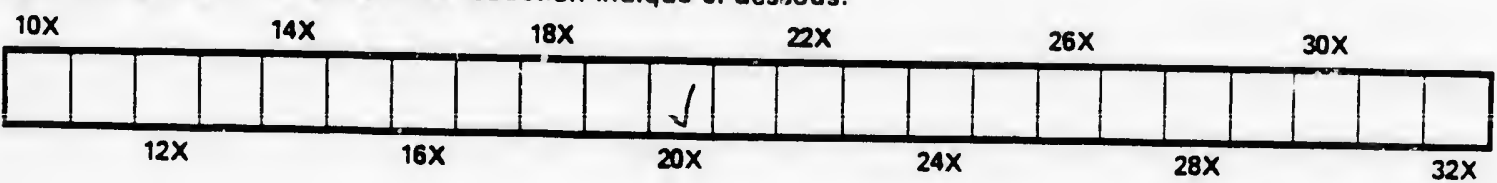


The copy filmed here has been reproduced thanks to the ganarosity of:

Library

Agriculture Canada

The images appearing here are the heat quaiity possibie considering the condition and legibility of the originai copy and In keeping with the fiiming contract apecifications.

Originei copies in printed paper covers are fiimed beginning with the front cover end ending on tha last pega with a printed or iiiustrated impression, or the beck cover when eppropriote. All other originai copies are fiimed beginning on the first page with a printed or iilustrated imprea. sion, and anding on the laat page with o printed or lliustreted impreaslon.

The leat recorded frame on each microfiche shail contain the symboi $\rightarrow$ Imeaning "CON TINUED"), or the symboi $\nabla$ (meening "END"). whichever appiies.

Meps, pletes, charts, otc., may be flimed at different reduction retioa. Those too large to be entireiy included in one axposure are fiimed beginning in the upper loft hend comer, ioft to right and top to bottom, as mony frames as required. The foliowing diegrema iilustrete the mothod:
L'exempiaire fiimd fut reproduit grace a la génórosito de:

Bibliothique

Agriculture Canada

Les images suivantes ont dté raproduites avec le pius grend soin, compte tenu de is condition ot de is nettete de l'axempiaire flimb, ot en conformitó avec les conditions du contrat de flimage.

Les exempiaires originaux dont la couverture en popier eat imprimbe sont fiimds en commencant per lo promier plet ot en terminent soit per la dernidre page qui comporte une ampreinte dimpression ou d'iilustration, soit par le second plat. selon le cas. Tous les autres exemplaires originaux sont flimbe on commencent par la premidre pege qui comporte une empreinte d'impression ou d'illustration et en terminent par ia dernidre page qui comporte une telie empreinte.

Un des symboies suivants epperaitra sup la dernidre image de chaque microfiche, selon le cas: is symboie $\longrightarrow$ signifle "A SUIVRE", le symboie $\nabla$ signifie "FiN".

Les cartes, pienches, tableaux, etc., peuvent atre flimbe a des taux de roduction différents. Lorsque le document est trop grend pour atre reproduit en un seui clicho. ii est fiimd a pertis de irangie supdrieur gauche, de gauche d droite, - de hout an bas, en prenent le nombre d'imeges ndcesseire. Les dlagrammes suivants iiiustrent le móthode.

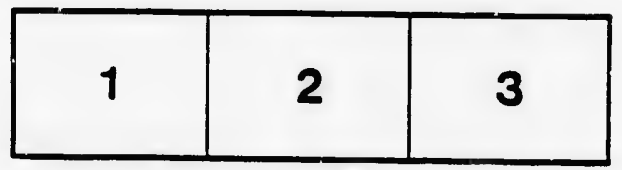

\begin{tabular}{|l|l|l|}
\hline 1 & 2 & 3 \\
\hline 4 & 5 & 6 \\
\hline
\end{tabular}

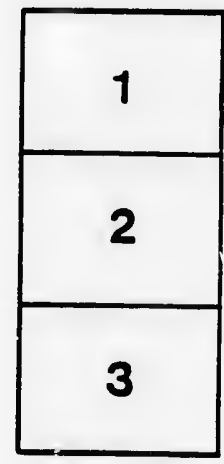





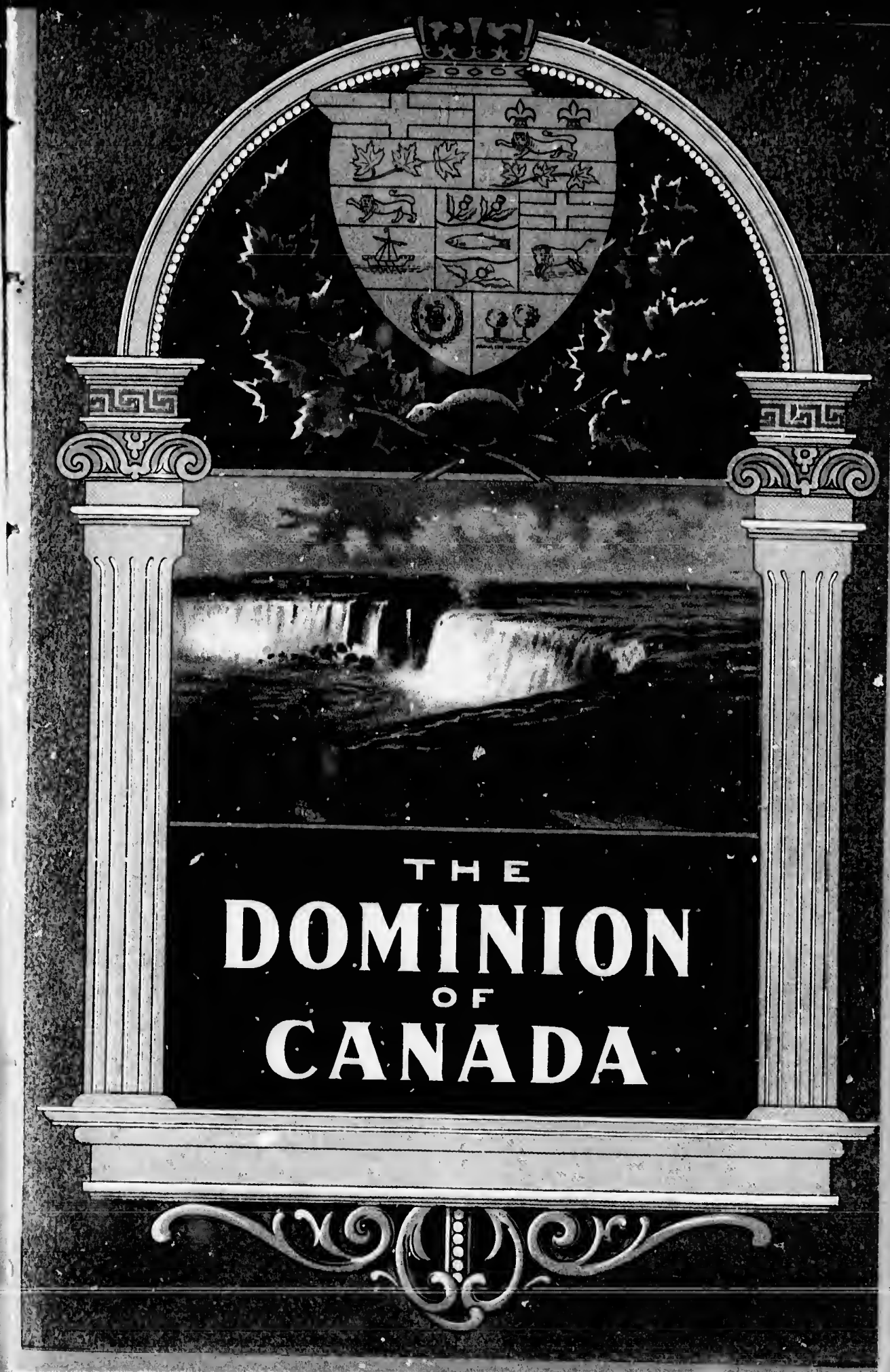





\section{FOREST, SPORT AND FISHING.}

\section{Dominion of Canada, Department of Marine and Minister. Professor Andrew. Gourdeau, Deputy Minister.}

\section{ALEOTION OF MAMMALS, BIRDS,}

Harbour Seal. Iè Callocephale veau Marin.

Phoca vitulina, L.

Gulf of St. Lawrence.

Harp Seal. Le Phoque Groenland.

Phoca groenlandica, Fabricius.

3 A specimen from Godbout, Province of Quebec.

4 Two young (or baby) specimens from Gulf of St. Lawrence.

5 Ringed Seal. Le Phoque a Collier.

Phoca foetida, Fabricius.

Two Specimens from Gulf of St. Lawrence.

6 Hooded Seal. Le Stemmatope à Casque.

Cystophora cristata, Erxleben.

Two large and one small specimens from Gulf of St. Lawrence.

7 Fur Seal. L'Otarie.

Callorhinus ursinus.

Two young specimens from Pacific Ocean.

8 Atlantic Walrus. Le Morse Cheval Marin.

Odobaeus rosmarus, Linnæus.

A specimen of head and a few tusks from Hudson Bay.

9 Two specimens of Ainerican Otter. La Loutre d'Amerique.

This species is cosmopolitall in Canada.

10 Three specimens of Mink. Le Vison d'Amerique.

This species is cosmopolitan in Canada. 
11 Fisher. Is Pécheur.

Muste'a pennunti, lirxleben.

One specimen from Ontario.

12 Musk Rat. L'Ondatra Musqué.

Fiber zibethicus, Linnens.

Three specimens from Ontario.

13 Benver. Lu Castor Fiber.

Castor canadensis, Kulll.

This species is cosmopolitan in Cunada.

14 Tusk of Narwhal. Le Narval Monoceros.

Monodon moniceros.

From Hudson Bay.

15 Moose Head. L'Elan d'Amerique.

Alce alces amerì zanus, Jardine.

COLLECTION OF BIRDS AND SMALL MAMMALS FROM PRINCE EDWARD ISLAND.

16 Mink. Le Vison d'Amerique.

Putorius vison, Schreber.

17 Red-fox. Le Renard Rouge.

Vulpes vulpes, Linnzeus.

18 Northern Hare. Le Liévre du Nord.

Lepus americanus virginianus, Harlan.

19 Common Loon. Le Plongeon a Collier.

Urinator imber, Gunner.

20 Great black backed Gull. Le Goeland a Manteau Noir.

Larus marinus, Linnæus.

21 American Herring Gull. Le Goeland Argenté.

Larus argentatus smithsonianus, Coues.

22 Common Tern. La Sterne de Wilson.

Sterna hirundo, Linnæus.

23 American Merganser. Le Harle d'Amerique.

Merganser americanus, Cass. 
24 Gadwall. Le Canard Chipenu. Anas strepera, Linnseus.

25 American Golden-eye. Le Bucéphale d'A merique. Glaucionetta clangula americana, lionaparte.

26 Surf Duck. Le Canard Macreuse i large Bec. Oidemia perspicillata, Linnieus.

27 Brant. La Bernache Commune. Branta bernicla, Linnæeus.

28 American Bittern. Le Butor. Botaurus lentiginosus, Montagu.

29 American Woodçock. La Bécasse d'Amerique Philohela minor, Ginelin.

30 American Golden Plover. Le Pluvier Doré, Charadrius dominicus, Müller.

31 Canada Ruffed Grouse. La Gélinotte it Fraise. Ronasa umbellus togata, Linnæus.

32 American Goshawk. L'Autour i tête Noire. Accipiter atricapillus, Wilson.

33 Red-shouldered Hawk. La Buse a Manteau Roux. Buteo lineatus, Gmelin.

34 American Sparrow riawk. Le Faucon"Epervier. Falco sparverius, Linnæus.

35 American Long-eared Owl. Le Hibou à Aigret.es Longues. Asio vilsonianus, Lesson.

36 Barred Owl. La Chouette du Canada. Syrnium nebulosum, Forster.

37 Great Horned Owl. Le Duc de Virginie. Bubc virginianus, Gmelin.

38 American Hawk Owl. La Chouette Eperviere. Surnia ulula caparoch, Muller.

39 Blue Jay. Ie Geai Huppé.

Cyanocitla cristata, Linnæus.

40 Cedar Bird. Ie Jaseur du Cèdre.

Ampelis cedrorum, Vieillot. 
41 Three specimens of young Ostriches and two eggs. L'Autruche Struthio camclus.

From Ostrich-farm near Toronto, Ontario. Joaned by Mr. Lundy through "R. Parker \& Co., Ostrich Fenther Dyers, Toronto."

42 Down of Américan Eider Dnck. L'Eider d'Anérique.

Somateria dresseri, Sharpe.

From one of the outer islands near Ste. Angustine River, Gulf of St. Lawrence.

\section{BIRDS' EGGS. GUFS D'OISEAUX. \\ CASE I.}

Tray 123.

43 Canada Goose. La Bernache du Canada.

Branta Canadensis, Linnieus.

A specimen from Morell, Prince Edward Island. Collected by Mr. Ernest Kemp.

Razor-billed Auk. Le Pingouin Commun.

Alca torda, Linnreus.

44 A specimen from Bird Rocks, Magdalen Islands. Collection of Mr. Rodolphe Lemieux, M.P.

Tray 124.

45 A specimen from Magdalen Islands. Collected by Mr. Camille Delaney.

Kittiwake Gull. La Mouette ì trois doigts.

Rissa-Tridactyla, Linnzeus.

46 Two specimens from Magdalen Islands. Collected by Mr. Camille Delaney.

Tray 132.

47 Two specimens from Bird Rocks, Magdalen Islands. Collection of Mr. Rodolphe Lemieux, M.P.

Murre. Le c'tillemot à ventre blanc.

Uria troile, Linr.aus.

Tray 125. Tray 126.

48 Four specimens from Magdalen Islands. Collected by Mr. Camille Delaney.

Tray 127. Tray 128.

49 Three specimens from Bird Rocks, Magdalen Islands. Collection of Mr. Rodolphe Lemieux, M.P.

White Gannet. Le Fou de Bassan.

Sula bassana, Linnæeus. 
Tray 128.

50 One specimen from Bird Rocks, Magdalen Islands. Collected by Tray 129.

51 Two specimens from Bird Rocks, Magdalen Islands. Collection Trays 130-131.

Great Northern Diver. Le Plongeon a Collier.

Urinator imber, Gunner.

52 Four specimens from Canadian Fishery Museum. This species of bird is cosmopolitan in Canada.

Tray 132.

53 Puffin. Le Macareux Arctique.

Fratercula arctica, Linnæus.

A specimen from Bird Rocks, Magdalen Islands. Collection of Tray 133

54 American Bittern. Le Butor.

Buiüïrus lentiginosus, Montague.

Three specimens from Long Point, Lake Erie.

55 Least Bittern: Le Petit Butor.

Botauris exilis, Gmelin.

Three specimens from Long Point, Lake Erie.

Tray 134.

Common Tern. Ia Sterne de Wilson.

Sterna hirundo, Linnæus.

56 Six specimens from Morell, Prince Edward Island.

Tray 135.

57 Three specimens from Tracadie, Nova Scotia. Collected by Mr.

Tray 135.

58 Black Tern. La Sterne Noire.

Hydrochelidon nigra surinamernsis, Gmelin.

Four specimens from Long Point, Lake Erie.

Tray 130.

59 Common Gallinule. La Gallinule de la Floride.

Gallinula galeata, Light.

Five specimens from Long Point, Lake Eric.

Piping Plover. Le Pluvier Criard.

Aegialitis meloda, Ord.

60 One specimen from Morell, Prince Edward Island. Collected by
Mr. Ernest Kemp. 
Tray 137.

61 Four specinens from Long Point, Iake Erie.

Tray 138.

62 Four specinens from Sable loland, Nova Scotia.

Tray 137.

63 Kill-Deer Plover. Le Pluvier Kildir.

Aegialitis vocifera, Linneus.

Four specimens from_Long Point, Lake Erie.

Trily 138.

64 Lenst Sand-piper. La Maubéclıe de Wilson.

Tringa minutilla, Vieillot.

Four specineıs from Sable Island, Nova Scotia.

CASE J.

Trays 139-154.

65 Murres' Eggs.

Uria troile, Linneus.

Sixteen specimens from Bird Rocks, Magdalen Islands. Collected by Commander William Wakeliam "La Canadienne."

\section{Province of Quebec, Department of Agriculture.}

Hon. F. G. M. Dechene, Minister, Quebec.

66 Moose. L'Elan d'Anerique.

Alce alces americanus, Jardine.

Specimens of male, female and calf.

67 Two specimens of Woodland Caribou or Reindeer. Le Renne Rangifer tarandus caribu, Kerr.

Caribou.

68 Red Deer. Ie Cerf de_Virginie.

Cariacus virginianus, Boddaert.

Two specimens of adults and a fawn.

69 Two specimens of Beaver. Le Castor Fiber.

Castor canadensis, Kuhl.

70 Black Squirrel. L'Ecureuil Noir

Sciurus carolinensis, Gmelin. 
71 Two specimens of Bluck benr. L'Onr Noir d'Ameriqun.

7.2 Specimen of Brown Bear. L'Ours Brun d'Aurerique.

Ursus americanus, Pallas.

Authorities differ as to this being a clistinct species from the
above.

73 Two specinens of Wolf. Ie Lou\}) Vulgaire.

Canis Lupus, Linnsens.

74 Two specimens of Esquimanx Dog. Le Chien des Esquinanx.

Musela americuna, Turton.

76 Two specimens of Skunk. Ie Zorille Varié.

Mephistis mephisticu, Shaw.

77 Specimen of Fisher. Le Fêcheur.

Mustela pennanti, Erxleben.

78 Specimen of Mink. Le Vison d'Amerique.

Putorius vison, Schreber.

79 Two specimens of Wolverine. Le Glouton Artique on Borial.

80 Two specimens of Otter. La Lontre d'Amerique.

Lutra canadensis, Turton.

81 Red Fox. Le Penard Ronge.

Vulpes vulpes, $\mathrm{L}$.

82 Cross Fox. Le Renard Croisé.

Vulpes decussalus.

83 Silver Fox. Le Renard Argenté.

Vulpes, sp.

84 Two specimens Canada Lynx.

Lynx canadensis, Desmarest.

913 Hon. F. G. M. Dechene, Quebec, Que.

85 Moose. L'Elan d'Amerique.

Alce alces americanus, Jardine.

86 One specimen Moose Head. L'Elan d'Amerique.

Alce alces americanus, Jardine. 
87 Two specimens Red Deer Heads. Le Cerf de Virginie. Cariac'us virginianus, Boddaert.

88 Two specimens Wapiti Heads. Le Cerf Wapiti. Cervus canadersis, Frxleben.

89 Four specimens Moose Heads. L'Elan d'Amerique. $\therefore$ 'ce alces americanvs, Jardine.

90 Five specimens Woodland Caribou Le Renne Caribou or Reindeer Heads. Rangifer tarandus caribu, Kerr.

91 One specimen Buffalo Head. Le Bieon d'Amerique. Bison americanus.

92 Two specimens Musk Ox Heads. L'Ovibos Musqué. Ovibos moschatus.

914 Sir Adolph Caron, Ottawa, Ont.

93 Une specimen Buffalo Head. Le Bison d'Amerique. Bison americanus.

915 D. R. Wilkie, Esq., Toronto, Ont.

94 One specimen Buffalo Head. Le Bison d'Amerique. Bison americanus.

916 J. R. Gill, Esq., Ottawa, Ont.

95 One specimen Moose Head. L'Elan d'Amerique Alce alces americanus, Jardine.

96 One specinen Wapiti Head. Le Cerf Wapiti. Cervus canadensis, Erxleben.

\section{FURS OF MAMMALS}

Inhabiting Anticosti Island, Fiuvince of Quebec.

917 Henry Menier. Through Dr. Schmitt.

97 Elack Bear. L'Ours Noir d'Amerique.

Ursus americanus, Pallas.

98 Red Fox. Le Renard Rouge.

Vulpes vulpes, Linnæus.

99 Silver Fox. Le Rénard Argenté.

Vulpes, sp. 
100 Cross Fox. Le Rénard Croisé.

Vulpes dicussatus.

101 Marten. La Marte Commune. Mustela americuna, Turton.

102 American Otter. La Loutre d'Amerique. Lutra canadeneis, Turton.

\section{FURS}

918 The Hudson Bay Company, London, England.

103 Common Raccoon. Le siaton Laveur. Procyon lotor, Linnæus.

104 Different varieties of the Black,

Brown, or Cinnamon Bear. ? L'Ours Noir d'Amerique. Ursus americanus, Pallas.

105 Polar Bear. L'Ours Blanc ou Polaire.

Thalarctos maritimus.

106 American Otter. Ia Loutre d'Amerique.

Lutra hudsonicu, Lacépède.

107 Skunk. Le Zorille Varie.

Mephitis mephitica, Shaw.

108 American Badger. Le Blaireau d'Amerique.

Taxidea americana, Boddaert.

109 Wolverine. Le Glouton Artique ou Boréal.

Gulo gulo, Lin næus.

110 Sable or Marten. La Marte Commine.

Mustela amiricana, Turton.

111 Mink. Le Vison d'Amerique.

Putorius vison, Schreber.

112 Ermine or Weasel. La Belette-Hermine.

Putorius erminea, Linnieus.

113 Red Fox (different varieties). Le Rénard Rouge.

Vulpes vulpes, Linnæus.

114 Silver Fox. Le Rénard Argenté.

Vulpes, sp.

115 Cross Fox. Le Rénard Croisé.

Vulpes aecussatus. 
116 Wolf. Le Loup Vulgaire.

Canis lupus, Linnæus.

117 Canada Lynx. Le Lynx du Canada.

Lynx canadensis, Desmarest.

118 Fisher. Le Pècheur.

Mustela pennanti, Erxleben.

119 Beaver. Le Castor Fiber.

Castor canadensis, Kuhl.

120 Northern Hare. Jue Liévre du Nord.

Lepus americanus virginianus, Harlan.

121 Musquash or Musk-rat. L'Ondatra Musqué.

Fiber zibethicus, Linnæus.

122 Musk Ox. L'Ovibos Musqué.

Ovibos muschatus.

919 G. E. Atkinson, Portage la Prairie, Manitoba.

123 Holboell's Grebe. Le Grébe a cou Rouge.

Colymbus holboellii, Reinhardt.

124 Horned Grebe. Le Grébe Cornu.

Colymbus auritus, Linnæus.

125 Pied-billed Grebe. Le Grébe à Bec Bigarré.

Podilymbus podiceps, Linnaus.

126 Common Loon or Great Northern Diver. Le Plongeon à Collier. Urinator imber, Gunner.

127 Franklin's Rosy Gull. La Mouette Rose de Franklin.

Larus franklinii, Swainson and Richardson.

128 Forster's Tern. La Sterne de Forster.

Sterna forsteri, Nuttall.

129 Common Tern or Wilson's Tern. La Sterne de Wilson.

Sterna hirundo, Linnæus.

130 Black Tern. La Sterne Noire.

Hydrochelidon nigra surinamensis, Gmelin.

131 Double-crested Cormorant. Le Cormoran ì Aigrettes.

Phalarrocorax dilophus, Swainson and Richardson.

132 Hooded Merganser. Le Petit Harle.

Lophodytes cucullatus, Linnæus. 
133 Mallard or Green Head. Le Canerd Ordinaire. Anas boschas, Linnreus.

134 Gadwall or Gray Duck. Le Canard Chipeau. Anas strepera, Linnæus.

135 Baldpate or American Widgeon. Ie Canard Siffleur d'Amerique.

136 Pin-tail Duck. Le Canard Pilet. Dafila acuta, Linnæus.

137 Wood Duck. Ie Canard Huppé. Aix sponsa, Linnæus.

138 Redhead. Le Canard Milouin a tête rousse. Aythya americana, Eyton.

139 Canvas-back. Ife Canard Milouin aux yeux rouges. Aythya vallisneria, Wilson.

140 Lesser Scaup Duck. Le Petit Morillon. Aylhyx affinis, Eyton.

141 Amerioan Golden-eye or Whistler. Le Bucéphale d'Amerique. Glaucionnetta clangula americana, Bonaparte.

142 Buffle-head. Le Petit Bucéphale. Charitonetta albeola, Linnæus.

143 Coween, Long-tailed Duck, or Old Squaw. Le Canard à Longue Clangula hyemalis, Linnæus. Queue.

144 Ruddy Duck. Le Canard Roux. Erismatura rubida, Wilson.

145 Blue Snow Goose. I'Oie du Nord. Chen corulescens, Linnæus.

Considered by some ornithologists to be a variety of the next mentioned. The French names L'Oie du Ford the next Blanche are applicable to both varieties.

146 Snow Goose. L'Oie Blanche.

Chen hyperborea, Pallas.

147 Blue-winged Teal. La Sarcelle à Ailes Bleues. Aras discors, Linnæus.

148 Shoveller. Le Canard Souchet. Spatula clypeata, Linnerus.

Speciunen of male. 
149 Canada Goose. La Bernache du Canada.

Branta canadensis, Linnæus.

150 American White-fronted Goose. L'Oie d'Amerique a Front Blanc. 'Anser albifrons gambeli, Hartlaub.

151 American Bittern. Le Butor.

Botaurus lentiginosus, Montagu.

152 Great Blue Heron. Le Grand Héron Blue. Ardea herodias, Linnæus.

153 Sandhill Crane. La Grue du Canada. Grus mexicana, Muller.

Some ornithologists consider this and the next to be the same species. Hence the name "La Grue du Canada" as applied to both.

154 Little Brown Crane. La Grue du Canada. Grus canadensis, Linnæus.

155 Virginia Rail. Le Rale de Virginie. Rallus virginianus, Linnæus.

156 Sora Rail or Ortolan. Le Rale de la Caroline. Porzana corolina, Linnæus.

157 American Coot. La Foulque d'Amerique. Fulica americana, Gmelin.

158 Wilson's Phalarope. Le Phalarope de Wilson. Phalaropus tricolor, Vieillot.

159 Pectoral Sandpiper. La Maubèche Tachetée. Tringa maculata, Vieillot.

160 Least Sand-piper. La Maubèche de Wilson. Tringa minutilla, Vieillot.

161 Sanderling. Le Sanderling. Calidris arenaria, Linnæus.

162 Marbled Godwit. La Barge Marbrée. Limosa fedoa, Linnæus.

163 Greater Yellow-legs. Le Chevalier Aboyeur. Totanus melanoleucus, Gmelin.

164 Lesser Yellow-legs. Le Petit Chevalier a Pieds Jaunes Totanus flavipes, Gmelin.

165 Spotted Sand-piper. La Maubèche Taclıetée. Actitis macularia, Linnæus. 
166 Willet. Le Chevalier Semipalmé.

Symphemia semipulmatu, Gmeliı.

167 Bartramian Sand-piper. La Maubèche i Longue Queue. Bartramia longicauda, Bechstein.

168 American Golden Plover. Le Pluvier Doré. Charadrius dominicus, Muller.

169 Killdeer Plover. Le Pluvier Kildir. Egialitis vociferu, Linnæus.

170 Spruce Partridge or Canada Grouse. Le Tetras du Canada. Derdragajus canadensis, Linnæus.

171 Willow Ptarmigan. Le Lagopède des Saules. Lagopus lagopus, Linnæus.

172 Ruffed Grouse. La Gélinotte ì Fraise. Bonasa umbellus Linneus.

173 Pinnated Grouse or Prairie Hen. La Gélinotte des Prairies. Tympanuchus amcricarus, Reichenbach.

174 Sharp-tailed Gıouse. La Gélinotte à Queue Aigue.

Pediocxtus phasianellus, Linnseus.

175 Mourning Dove. La Tourterelle de la i'aroline. Zenaidura macroura, Linneus.

176 Turkey Vulture. Le Vautour Aura. Cathartes aura, Linneus.

177 Marsh Hawk or Harrier. Le Busard des Marais. Circus hudsonius, Linnzeus.

178 Sharp-shinned Hawk. L' Kpervier Brun. Accipiter velox, Wilson.

179 American Goshawk. L'Autour a Tête Noire. Accipiter airicapillus, Wilson.

180 Red-tailed Hawk. Ia Buse à Queue Rousse. Buteo borealis, Gmelin.

181 Swainson's Hawk. La Buse de Swainson. Buteo swainsoni, Bus arte.

182 Broad-winged Hawk. La Buse de Pennsylvanie. Buteo latissimus, Wilson.

183 Rough-legged Hawk. La Buse Pattue d'Amerique. Archibuteo lagopus, Brunnich. 
184 Golden Eagle. L'Aigle du Canada. Aguila chrysaetos, Linnæus.

185 Bald Eagle. L'Aigle a Tête Blanche.

Halivetus leucocephalus Linnrelis.

186 Peregrine Falcon or Duck Hawk. Le Faucon Pelerin.

Falco peregrinus anatum, Bonaparte.

187 American Long-eared Owl. Le Hibou i Aigrettes longues. Asio wilsonianus, Lesson.

188 Short-eared Owl. Le Hibou a Aigrettes Courtes. Asio accipitrinus, Pallas.

189 Barred Owl. La Chouette du Canada. Syrnium nebulosum, Forster.

190 Great Grey Owl. La Chouette Cendrée. clula cinerea, Gmelin.

191 Richardson's Owl. La Nyctale de Richardson. Nyctala tengmalmi richardsoni, Bonaparte.

192 Saw-whet Owl or Arcadian Owl. La Nyctale d'Arcadie. Nyctala acadica, Gmelin.

193 Burrowing Owl. Le Pheleoptynxdes Hypogées. Speotyto cunicularia hypogaea, Bonaparte.

194 Western Horned Owl. Le Duc de Virginie. Bubo virginianus subarcticus, Hoy.

195 Snowy Owl. Le Harfang.

Nyctea nyctea, Linnaeus.

Two specimens, male and female.

196 American Hawk Owl or Day Owl. La Chouette Eperviere. Surnia ulula caparoch, Muller.

197 Black-billed Cuckoo. Le Coucou à Bec Noir. Coccyzus erythrophthalmus, Wilson.

Two specimens and nest.

198 Belted Kingfisher. Le Martin Pêcheur.

Ceryle aicyon, Linnaeus.

199 Hairy Woodpecker. Le Pic Chevelu.

Dryobates villosus, Linnaeus.

200 Downy Woodpecker. Le Pic Minule.

Dryobates pubescens, Linnaeus. 
201 Arctic or Black-backed three-toed Woodpecker. Is Pic A retıque.

Picoides arcticus, Swainson.

202 Yellow-bellied Woodpecker. Le Pic Maculé.

Sphyra picus varius, Linnaeus.

203 Pileated Woodpecker. La Pic à Huppe ècarlate.

Ceophloeus pileatus, Linnaeus.

204 Red-headed Woodpecker. Le Pic il tete Rouge.

Melanerpes erythrocephalus, Linnaeus.

205 Flicker, Golden-wingedWoodpecker or High-holder. Le Pic Doré. Colaptes auratus, Linnaeus.

Two specimens (One a hybrid).

206 Chimney Swift. Le Martinet des Cheminées.

Chcetura pelagica, Einnæus.

207 Ruby-throated Humming Bird. Le Colibri ì gorge rubis.

Trochilus colubris, Linnæus.

208 Kingbird or Bee-martin. Le Tyran de la Caroline.

Tyrannus tyrannus, Linnæus.

209 Crested Flycatcher. Le Moucherolle à Huppe.

Myiarchus crinitus, Linnæus.

210 Phoebe. Le Moucherolle Brun.

Sayornis phoebe, Latham.

211 Olive-sided Flycatcher. Le Mouclierolle à cotes Olive.

Contopus borealis, Swainson.

212 Wood Pewee. Le Moucherolle verdatre.

Contopus virens, Linnæus.

213 Yellow-bellied Flycatcher. Le Moucherolle ì ventre jaune.

Empidonax flaviventris, Baird.

214 Traill's Flycatcher. Le Moucherolle de Trail.

Empidonax pusillus traillii, Audubon.

215 Least Flycatcher. Le petit Moucherolle.

Empidonax minimus, Baird.

216 Horned Lark. L'Alouette'Ordinaire ou de Virginie.

Otocoris alpestris, Linnæeus.

217 American Magpie. La Pie de la Baie d'Hudљon.

Pica pica hudsonica, Sabine. 
218 Blue Jay. Le Geni Hıppí.

Cyanocitta cristata, Limneus.

219 Canada Jay or Whisky Jack. Le Geai dı Canada.

Perisoreus canadensis, Linnaus.

220 Northern Raven. Ie Corbeau du Nord.

Corvus corax principalis, Ridgway.

221 American Crow. La Corneille d'Amerique. Corvus americanus, Audubon.

222 Bobolink. Le Goglu.

Dolichony $x$ oryzivorus, Tinnzeus.

223 Yellow-headed Blackbird. L'Etournean ì tete jaune.

Xanthocephalus xanthocephalus, Bonaparte.

Three specimens and nest.

224 Red-and-buff-shouldered Blackbird. L'Etourneau à ailes rouges. Agelaius phoeniceus, Linneus.

225 Meadow Lark. L'Etourneau des Prés.

Sturnella magna, Linnzeus.

226 Baltimore Oriole. L'Oriole de Baltimore.

Icterus galbula, Linnzeus.

227 Rusty blackbird or Grackle. Le Mainate Couleur de rouille.

Scolecophagus carolinus, Muller.

228 Brewer's Blackbird. La Mainate de Brewer.

Scolecophagus cyanocephalus, Wagler.

229 Evening Grosbeak. Le Gros-Bec à Couronne Noire.

Coccothraustes vespertina, Cooper.

230 American Pine Grosbeak. Le Bourreuil du Canada.

Pinicola enucleator canadensis, Cabanis.

231 Purple Finch. Le Pinson Pourpre.

Carpodacus purpureus, Gmelin.

232 American Red-cross bill. Le Bec-croisé d'Amerique.

Loxia curvirostra minor, Brehm.

233 White-winged crossbill. Le Bec-Croisé à ailes Blanches.

Loxia leucoptera, Gmelin.

234 Hoary Redpoll. Te Sizerin Blanchatre.

Acanthis hornemannii exilipes, Coues. 
235 Redpoll. Le Sizerin it tete rouge.

Acanthis linaria, Linneus.

236 American Goldfinch. Le Chardonneret jaune.

Spinus tristis, Linnieus.

237 Pine Siskin. Le Chardonneret des Pins.

Spinus pinus, Wilson.

238 Snow Bunting. Le Plectrophane des Neiges.

Plectrophenax nivalis, Linnæus.

239 Lapland Longspur. Le Plectrophane de Laponie.

Calcarius lapponicus, Linnzeus.

240 Chestnut-collared Longspur. Le Plectrophane.

Calcarius ornatus, Townsend.

241 Vesper :Sparrow or Gray-winged Bunting. Le Pinson des Poocaetus gramineus, Gmelin.

[Guérets.

242 Savannah Sparrow. Le Pinson de Savanna.

Ammodramus sandwichensis savanna, Wilson.

243 Le Conte's Sparrow. Le Pinson de Le Conte. Ammodramus leconteii, Audubon.

244 Lark Sparrow. Le Pinson a Oreilles Brunes.

Chondestes grammacus, Say.

245 Harris's Sparrow. Le Pinson de Harris.

Zonotrichia querula, Nuttall.

246 White-crowned Sparrow. Le Pinson a Couronne Blanclse .

Zonotrichia leucophrys, Forster.

247 White-throated Sparrow. Le Pinson a Gorge Blanche.

Zonotrichia albicollis, Gmelin.

248 Intermediate Sparrow. Le Pinson intermédiaire.

Zonotrichia intermedia, Ridgway.

249. Tree Sparrow. Le Pinson de Montagne.

Spizella monticolu, Gmelin.

250 Chipping Sparrow. Le Petit Pinson a Couronne Rousse. Spizella socialis, Wilson.

251 Clay-coloured Sparrow.

Spizella pallida, Swainson. 
252 Slate-coloured Junco. te Pinson Niverolle. Junco hyemalis, Linnens.

253 Lincoln's Sparrow. Lo Pinson de Lincolı. Melospiza lincolni, Andubon.

254 Swamp Sparrow. Le I'inson des Marais. Melorpiza georgiana, Latham.

255 Fox Sparrow. Ie Pinson Fauve. Passerella ilinca, Merrem.

256 Towlree. Le Pinson aux Yeux Ronges. Pipilo erythrophthalmus, Linnaeus.

257 Rose-Breasted Grosbenk. Ie Gros-Bec ì Poitrine Rose. Habia ludoviciana, Linnıens.

258 Scarlet Tanager. Le Tangara Ecarlate. Piranga erylhromelas, Vieillot.

259 Cliff Swallow. L'Hirondelle it Front Blanc. Petrochelidon lunifrons, Say.

260 Barn Swallow. L'Hirondelle des Granges. Chelidon erythrogaster, Boddaert.

261 Bolemian Waxwing. Le Jaseur de Bolıcme. Ampelis gamulus, Liunieus.

262 Cedar Waxwing. Le Jaseur du Cèdre. Ampelis cedrorum, Vieillot.

Three specimens and nest.

263 Northern Slırike or Butcher Bird. Le Pie-Grièche Boréale. Lanius lorealis, Vieillot.

264 White-rumped Shrike. La Pie-Grièche ì Croupion Blanc. Lanius ludoricianus excubitorides, Swainson.

265 Red-eyed Vireo. Le Vireo aux Yeux Ronges. Vireo olivuceus, Linnreus.

266 Philadelpluia Vireo. Le Vireo de Philadelphie. Vireo philadelphicus, Cassin.

267 Warbling Vireo. Le Vireo Gris. Vireo gilvus, Vieillot.

268 Blne-headed Vireo. Le Vireo Solitaire. Vireo solitarius, Wilson. 
269 Black and White Warbler. Io Grimpereau Noir ef Blauc. Mniotilta varia, Linnfeus.

270 Nashville Warbler. Ia Fauvette de Nashville. Helminthophila ruficapilla, Wilson.

271 Orange-crowned Warbler. La Fauvette a Couroure orange. Helminthophila celuta, Say.

272 Tennessee Warbler. La Fauvette du Tenuessee. Helminthophila peregrina, Wilyon.

273 Yellow Warbler. Ia Fauvette jaune.

Dendroica aestiva, Gmelin.

Two specimens and nest.

274 Myrtle Warbler. La Fauvette ì Croupion jaume.

Dendroica coronata, Limnieus.

275 Magnolia Warbler. La Fauvette ì tête cendrée. Dendroica maculosa, Ginelin.

276 Chestnut-sided warbler. La Fauvette de Pensylvanie. Dendroica pensylvanica, Linueus.

277 Bay-breasted Warbler. La Fauvette a Poitrine Baie.

Dendroica castanea, Wilson.

278 Black-poll Warbler. La Fauvette Rayée.

Dendroica striata, Forster.

279 Blackburnian Warbler. La Fauvette de Blackburn.

Dendroica blackburniae, Gmelin.

280 Palm Warbler. La Fauvette i tête Rousse.

Dendroica palmarum h!pochrysea, Ridgway.

281 Oven Bird. Ia Grive Couronnée.

Seiurus aurocapillus, Linuzeus.

282 Water Tlirush. La Grive Hochequene.

Seiurus noveboracensis, Gmelin.

283 Mourning Warbler. La Fauvette de Pliladelphie.

Geothlypis philadelphiı, Wilson.

284 Maryland Yellow-throat. La Fauvette Triclras. Geothlypis trichus, Liunieus.

285 Canadian Warbler. La Fauvette Moucherolle du Canada. Sylvania canadensis, Linnæeus. 
286 Anericu Redstart. Ia Fauvette Moucherolle Dorí. Setophaga ruticilla, Limneus.

287 Cat-bird. Ia Grive de la Caroline. Galeoscoptes carolinensis, Limmeus.

Two specimens and nest.

288 Brown Thrasher. La Grive Rousse. Harporhynchus rufus, Linneus,

289 House Wren. Is Troglodyte Adon.

Troglodytes aedon, Vieillot.

290 Winter Wren. Lo Troglodyte d'Hiver, Troglodytes hiemalis, Vieillot.

291 Short-billed Marsh Wren. Lo Troglodyte des Marnis. Cistothorus stellaris, Lichtenstein.

292 Brown Creeper. Lo Grimpereau d'Amérique. Gerthia familiaris americana, Bonaparte.

293 White-Breasted Nuthatch. La Sittelle de la Caroline. Silta carolinensis, Latham.

294 Red-breasted Nuthatch. La Sitelle du Cunada.

Silta canadensis, Linnaus.

295 Chickadee. La Mésange à tête Noire. Parus alricapillus, Linnreus.

296 Golden-crowned Kinglet. Le Roitelet Huppé. Regulus satrapa, Lichtenstein.

297 Ruby-crowned Kinglet. Io Roitelet i Couronne Rubis. Regulus calendula, , Linnacus.

298 Wilson's Thrush. La Grive de Wilson. Turdus fuscescens, Stepliens.

299 Gray-cheeked Thrush. La Grive aux Côtés de la tête Grisitres. Turdus alicice, Baird.

300 Olive backed Thrush. La Grive de Swainson.

Turdus ustulatus swainsonii, Cabanis.

301 Hermit Thrush. Inive Oniitaire.

Turdus sco, hat sce pallasii, Cabanis.

302 Blue Bird. Le Ronge-gorge Bleu.

Sialia sialis, I.inneus. 
303 Moose Heads. I'Elan d'Amérique.

Alce alces americanus, Jardine.

920 Alox. Oaldor, Irimnipeg, Manituba.

304 Western Grebe. Io Gribe Occidentalis.

Achmophorus occidentalis, Lawrence.

305 American Red-necked Grebe, or Holboell's Grebe. Lo Grìbe a Colymbus holboellii, Reinhardt.

[Cou Rouge.

306 Horned Grebe. Ie Grèbe Cornu.

Colymbuy auritus, Linnzeus.

307 Crested Grebe. Le Grùbe Huppé.

Podicipes cristatus, Iatham.

308 Great Northern Diver or Common Loon. Le Plongeon a Collier. Urinator imber, Gunner.

309 Double Crested Cormorant. Le Cormoran d'Aigrettes.

Phalacrocorax dilophus, Swainson and Richardson.

310 American White Pelican. Le Pélican Blanc.

Pelecanus erythrurhynchos, Gmelin.

311 American Merganser or Sheldrake. Le Harle d'Amérique.

312 Hooded Merganser. Lo Petit Harle.

Lophodyles cucullatus, Linnsus.

313 Mallard or Greenhead. Le Canard Ordinaire.

Anas boschus, I.innæus.

314 American Widgeon or Baldpate. Lo Canard, Siffleur d'AmèriAnas am ricana, Gmelin. [que.

315 Green-winged Teal. La Sarcelles i Ailes Vertes. Anas carolinensis, Gmelin.

316 Blue-winged Teal. La Sarcelle a Ailes Bleues. Anas discors, Linnseus.

317 Pin-tail Duck. Le Canard Pilet. Dafila acula, Linnæus.

318 Wood Duck. Le Canard Huppé. Aix sponsa, Linnaus. 
319 American Pochard or Red-head. Le Canard Milouin a tête Aythya americana, Eyton.

[Rousse.

3:0 Canvas-back Duck. Le Canard Milouin aux Yeux Rouges. Aythya vallisneria, Wilson.

321 American Scaup Duck. Le Morillon ì tête Noire. Aythya marila nearctica, Stejneger.

322 Buftle-head. Le Petit Bucephale.

Churitonitta albeola, Linnseus.

323 Laughing Goose. L'Oie d'Amérique a Front Blanc. Anser albifrons gambeli, Hartl.

324 Canada Goose. La Bernache du Canada. Branta canadensis, Linnceus.

325 Black-crowned Night Heron. Le Héron de Nuit. Nycticorax nycticorax nevius, Boddaert.

326 White or Whooping Crane. La Grue d'Amérique. Grus americana. Linnseus.

327 Wiloun's Phalarope. Te Phalarope de Wilson.

Phalaropus tricolor, Vieillot.

$328 \mathrm{Marl}^{\prime} \mathrm{n}$ or Great Marbled Godwit. La Barge Marbrée.

Limosi fedoa, Linnseus.

329 Dusky Grouse.

Dendragapus obscurus, Say.

330 Ruffed Grouse. La Gélinotte ì Fraise.

Bonasa umbellus, Linnæus.

331 Rock Ptarmigan. Le Lagopède des Rochers.

Lago pus rupestris, Gmelin.

332 Pinnated Grouse or Prairie Hen. La Gélinotte des Prairies. Tympanuchus americanus, Reichenbach.

333 Golden Eagle. L'Aigle du Canada.

Aquila chrysielos, Linnieus.

334 Bald Eagle. L'Aigle à Tête Blanche.

Haliretus leucocephalus, Linneus.

335 Duck Hawk or Peregrine Falcon. Le Faucon Pélerin.

Falco persgrinus anatum, Bonaparte. 
336 Western Barred Owl. La Chonnette Occidentale. Syrnium occidentale, Xantus.

337 Screech or Mottled ()wl. Ie Scops Maculé.

Megascops asio, Linneus.

338 Great Horned Owl. Le Duc de Virginie.

Bubo virginianus, Gmelin.

339. Snowy Owl. Le Harfang.

Nyclea nyctea, Linnæus.

340 Burrowing Owl. Le Pheleoptynxde Hypodées.

Speotyto cunicularia hypogr', Bonaparte.

341 Black-tail Deer Head.

C'ervus columbianus.

342 Wapiti Heads. Le Cerf Wapiti.

Crrvus canadensis, Erxleben.

343 Moose Heads. L'Elan d'Amerique.

Alce alces americanus, Jardine.

344 Woodland Caribou or Reindeer Head. Le Renne Caribou.

Rangifer tarandus caribu, Kerr. 345 Rocky Mountain Antelope Head. Le Capricorne d'Amerique.
Antilocipra americana, Ord.

346 Buffalo Heads. Le Bison d'Amerique.

Bis $n$ americanus.

347 Musk Ox Head. L'Ovibos Musqué.

Ovibos moschalus.

921 John Perrett, Sherbrooke, Province of Queluec.

A.-Group of Water Birds in a case of rock work.

B.-A case of perching, climbing, and ground birds.

C. - Group of Birds in winter plumage.

D. - Birds mounted singly upon stands.

E.-Mammals mounted singly upon stands and Mamianl Heals, 


\section{(A) GROUP OF WATER BIRDS.}

348 Horned Grebe. Le Grébe Cornu.

Colymbus auritus, $\mathrm{I}$.

349 Pied-billed Grebe (male and female). Ia Grébe a Bec Bigarré. Podilymbus podiceps, $\mathrm{L}$.

350 Great Northern Diver. Le Plongeon a Collier Jrinator imber, Gunn.

351 Red-throated Loon. Le Plongeon du Nord. Urinator lumme, Gunn.

352 Black Guillemot. Le Guillemist Noir.

Cepphus grylle, $\mathrm{L}$.

353 Razor-billed Auk. Le Pingouin Commun. Alca torda, $\mathrm{L}$.

354 Long-tailed Jaeger. Le Stercoraire de Buffon. Stercorarius longicaudus, Vieillot.

355 Ivory Gull. La Mouette Blanche.

Gavia alba. Gunn.

356 Kittiwake Gull. La Mouette à Trois Doigts. Rissa tridactylı, I.

357 American Herring Gull. Le Goeland Argenté. Larus argentutus smithsonianus, Coues.

358 Bonaparte's Gull. Le Mouette de Bonaparte. Larus philadelphia, Ord.

359 Amarican Merganser (female and young male). Le itarle d'AmerMerganser americanus, Cass.

360 Black Duck (male). Le Canard Noir. Anas obscura, Gmelin.

361 American Widgeon (young male). Le Canard siffleur d'Amerique. Anas americana, Gmelin.

362 Green-Winged Teal (male). Le Sarcelle ì ailes vertes. Anus carolinensis, Gmelin.

363 Pin-tail (male). Le Canard Pilet. Dafilu ac uta, L.

36t Wood-duck. Le Canard Huppé. Aix sponsa, $\mathrm{L}$. 
365 Red-head (male). Le Canard Milouin ì tête Rousse. Aythya americana, Eyt.

366 Canvas-back Duck (male). Le Canard Milouin aux yeux Rouges. Aythya vallisneria, Wils.

367 American Golden-eye. Le Bucéphale d'Amerique. Glaucionetta clangula americana, Bonap.

368 Old Squaw, (male.) Le Canard a Longue Queue.
Clangula hyemalis, L. 369 American Black Scoter (male). Ite Canard Macreuse d'Amerique.
Oidemia amsricana, Sw. and Rich.

370 Ruddy Duck (female). Le Canard Roux. Erismatura rubida, Wils.

371 Canada Goose. La Bernache du Canada. Branta canaderisis, L.

372 American Bittern. Le Butor. Botaurus lentiginosus, Montag.

373 Least Bittern. Le Petit Butor. Botaurus exilis, Gmel.

374 King Rail. Le Raleéi egant. Rallus elegans, Aud.

375 Virginia Rail. Le Rale de Virginie.

Rallus virginianus, $\mathrm{L}$.

376 Wilson's Snipe (male and female). La Becassine de Wilson. Gallinago delicata, Ord.

377 Least Sand-piper. La Maubèche de Wilson. Tringa minutilla, Vieill.

378 Red-backed Sand-piper. La Maubèche à Dos roux. Tringa alpina pacifica, Coues.

379 Greater Yellow-shanks. Le Chevalier Aboyeur. Totanus melanoleucue, Gmel.

380 Least Yellow-shanks. Le Petit Chevalier i Pieds Jaunes. Totanus flavipes, Ginel.

381 Ring-necked Plover. Ie Pluvier semipalmé. Agialitis hiaticula, $\mathrm{L}$. 
382 Wilson's Plover. Le Pluvier de Wilson.

Agialitis wilsonia, Ord.

383 Belted Kingfisher. Le Martin Pêcheur.

Ceryle alcyon, $\mathbf{L}$.

(B). GROUP OF PERCHING, OLIMBING, AND GROUND BIRDS.

384 Virginia Rail. Le Rale de Virginie.

Railus virginianus, $\mathbf{L}$.

385 Wood-cock (male and female). La Bécasse d'Amerique.

Fhilohela minor, Gmel.

386 Wilson's Snipe (two males). La Bécassine de Wilson.

Gallinago de'ícata, Ord.

387 Solitary Sand-piper. Le Chevalier Solitaire.

Totanus solitarius, Wils.

388 Canada Grouse (male and female). Ite Tétras du Canada. Dendragapus canadensis, $\mathrm{L}$.

389 Ruffed Grouse (male). Le Lagopède des Saules.

Bonasa umbellus, $\mathbf{L}$.

390) Northern sharp-tailed Grouse. La Gelinotte à Queue Aigue. Pediocietes phasianellus, Linnæus.

391 Sliarp-shined Haw's (male). L'Epervier Brun. Accipiter yelox, Wils.

392 Red-shouldered Hawk. La Buse a Manteau Roux.

Buteo iineatus, Gmel.

393 Pigeon Hawk (male). Le Faucon des Pigeons.

Falco columbarius, L.

394 American Sparrow Hawk (male). Le Faucon Epervier. Falco sparverius.

395 American Loug-eared Owl. Le Hibou à Aigrettes Longues. Asio wilsonianus, Less.

396 Short-eared Owl. Le Hibou ì Aigrettes Courtes.

Asio accipitrinus, Pall.

397 Barred Owl (with Weasel). La Chonette du Canada.

Sirnium nelulosum, Forst. 
398 Richardson's Owl. Ia Nyctale de Richardson.

Nyctala tengmalmi richardsoni, Bonap.

399 Saw-whet or Acadian Owl. La Nyctale d'Acadie.

Nyctala acadica, Gmel.

400 Great Horned Owl imale and female). Le Duc de Virginie.

Bubo virginianus, Gmel.

401 American Hawk Owl. La Chouette Eperviere.

Surnia ulula caparoch, Mull.

402 Yellow-billed Cuckoo. Le Coucou d'Amerique.

Coccyzus americanus, $\mathrm{L}$.

403 Belted Kingfisher. Le Martin-Pêcheur.

Ceryle alcyon, $\mathrm{I}$.

404 Hairy Woodpecker (male). Le Pic Chevelu.

Dryobates villosus, $\mathrm{L}$.

405 Downy Woodpecker (male). Le Pic Minule.

Dryobates pubescens, L.

406 Black-backed, three-toed Woodpecker (male and female).

Picoides arcticus, Swains. Le Pic Arctique.

407 Banded three-toed Woodpecker (male and female). Le Pic Velu. Picoides americanus, Brehm.

408 Yellow-bellied Woodpecker (male and female) Le Pic Macule. sphyrapicus varius, I.

409 Pileated Woodpecker (male). Le Pic à Huppé ecarlate.

Ceophlous pileatus, L.

410 Yellow Hammer or Flicker (male). Le Pic Doré.

Colaptes auratus, L.

411 Chimney Swift. Le Martinet des Cheminées.

Chetura pelagica, L.

412 Ruby-throated Humming Bird. Le Colibri ì Gorge Rubis.

Trochilus colubris, L.

413 Bee-martin or King Bird. Le Tyran de la Caroline.

Tyrannus tyrannus, L.

414 Phobe. Le Moucherolle Brun.

Sayornis Phebe, Lath. 
415 Wood Pewee. Le Moucherolle Verdatres.

Contopus virens, $\mathbf{L}$.

416 Blue' Jay. Le Geai_Huppé.:

Cyanocitta cristata, $\mathrm{L}$.

417 Canada Jay. Le Geai du Canada.

Perisoreus canadensis, $\mathrm{L}$.

418 American Crow. La Corneille d'Amerique.

Corvus ame: icanus, Aud.

419 Bobolink. Le Goglu.

Dolichonyx oryzivorus, $\mathrm{L}$.

420 Cow Bird. L'Etourneau Ordinaire. Molothrus ater, Bodd.

421 Red-and-buff-shouldered Black Bird (male). L'Etourneau à ailes Agelaius phoniceus, L. Rouges.

422 Baltimore Oriole. L'Oriole de Baltimore.

Icterus galbula, L.

423 American Pine Grosbeak. Le Bouvreuil dı Canada.

Pinicola enucleator canadensis, Cab.

424 American Red Crossbill. Le Bec Croisé d'Amerique.

Loxia curvirostra minor, Brehm.

425 White-winged Crossbill (:aale and female). Le Bec Croisé à ailes Loxia leucoptera, Gmel. Blanches.

426 European House Sparrow. Le Moineau Domestique.

Passer domesticus, L. (An introduced species).

This specimen is of the nature of an albino, being a uniform light gray colour.

427 Vesper Sparrow. Le Pinson des Guérets.

Poocates gramineus, Gmel.

428 White-crowned Sparrow. Le Pinson a Couronne Blanche.

Zonotrichia leucophrys, Forst.

429 Yellow-winged Sparrow. Le Pinson a Ailes Jaunes.

Ammodramus savannarum passerinus, Wilson.

430 Tree Sparrow. Le Pinzon de Montagne.

Spizella monticola, Gmel. 
431 Chipping Sparrow. Le Petit Pinson a Couroune Rousse. Spizella socialis, Wils.

432 Field Sparrow. Is Pinson des Champs.

Spizella pusilla, Wils.

433 Rose-breasted Grosbeak. Le Gros Bec a Poitrine Rose.

Halria ludoviciana, $\mathrm{L}$.

434 Indigo Bunting. Le Pinson Indigo.

Passerina cyanea, L.

435 Scarlet Tanager. Le Tangara Ecarlate.

Piranga erythromelas, Vieill.

436 White-bellied Swallow. L'Hirondelle Bicolore.

Tach? cineta bicolor, Vieill.

437 Cedar Wax-wing. Le Jaseur du Cèdre.

Ampelis cedrorum, Vieill.

438 Vireo. Le Vireo.

Vireo, sp.

439 Blue Yellow-backed Warbler. La Fauvette d'Amerique.

Compsothlypis amıricana, $\mathrm{L}$.

440 Cape May Warbler. La Fauvette du Cap May.

Dendroica tigrina, Gmel.

441 Summer Yellow Bird. La Fauvette Jaune.

Dendroica rstiva, Gmel.

442 Yellow Rumped Warbler (male and female).

Dendroica coronata, $\mathrm{L}$.

La Fauvette a Croupion Jaune.

443 Magnolia Warbler. La Fauvette i tete Cendrée.

Denilroica muculosa, Gmel.

444 Chestnut-sided Warbler (male and female). La Fauvette de

Pennsylvanie.

Dendroica pensylvanica, $\mathrm{L}$.

445 Blackburnian Warbler (male and female). La Fauvette de Dendroica blackburnia, Gmel.

Blackburn.

446 Black-throated Green Warbler. La Fauvette Poitrine Noire. Dendroica virens, Gmol.

447 Maryland-yellow-throat. La Fauvette Trichas.

Geothlypis trichas, $\mathrm{L}$. 
448 Black-capped Warbler. Ia Fauvette de Wilson.

Sylvania pusilla, Wils.

449 American Redstart. La Fauvette Mouclerolle Doré.

Setophaga ruticilla, I.

450 Cat Bird. Le Grive de la Caroline.

Galeoscoptes carolinensis, I.

451 House Wren. Le Troglodyte Adon.

Troglodytes aedon, Vieill.

452 Wood Thrush. La Grive des Bois.

Turdus mustelinus, Gmel.

453 Blue Bird. Le Rouge Gorge Blue.

Sialia sialis, I.

(C.) GROU'P OF BIRDS IN WINTER PLUMAGE.

454 Two Willow Ptarmigans. Le Lagopède des Saules.

Lagopus lagopus, $\mathrm{L}$.

455 Great Gray Owl. 'a Chouette Cendrée.

Ulula cinerea, Gmel.

456 Snowy Owl. Le Hat fang.

Nyctea nyctea, L.

457 Three Snow Buntings. Le Plectrophane des Neiges.

Plectrophenax nivalis, $\mathrm{L}$.

(D). BIRDS MOUNTED SINGLY UPON STANIS.

458 Horned Grebe. Le Grebe Cornu.

Colymlus auritus, $\mathrm{L}$.

459 Canadian Ruffed Grouse (male and female). Ia Gélinotte dı Bonasa umbellus togata, $\mathrm{L}$. Canada.

460 Sharp-shinned Hawk. L'Epervier Brun. Accipiter velox, Wils.

461 Cooper's Hawk. L'Epervier de Cooper. Accipiter cooperi, Bonap.

462 American Gos Hawk. L'Autour a tete Noire. Accipiter atricapillus, Wilson.

463 Red-tailed Inwk. Ia Buse à Queue Rousse. Buteo borealis, Gmel. 
464 Red-shouldered Hawk. La Buse a Mantomu lioux. Buteo lineutu", Ginel.

465 American Osprey. Le Balbusard de la Caroline. Pandion haliaetus carolinensis, Ginel.

4 (i6 American-long-eared Owl. Le Hibou i Aigrettes longues. Asio wi'sonianus, Less.

467 Short eared Owl. Le Hibou a Aigrettes Courtes. Asio accipitrinus, Pall.

468 Richardson's Owl. Le Nyctale de Richardson.

Nyctala tengmalmi richardsoni, Bonap.

469 Saw-whet or Acadian Owl. La Nyctale d'Acadie. Nyctala acadica, Gmel.

470 Great-horned Owl. Le I uc de Virginie.

Bubo virginianus, Gimel.

471 American Hawk Owl. La Chouette Eperviere.

Surnia ulula caparoch, Mull.

472 King Bird or Bee Martin. Le Tyran de la Caroline.

Tyrannus tyrannus, L.

473 Phoebe. Le Moucherolle Brun.

Sayornes phoebe, Lath.

474 Canada Jay or Whisky Jack. Le Geai du Cauada.

Perisoreus canadensis, L.

475 Butcher Bird or Great Northern Shrike. La Pie Grieche Boreale. Lanius borealis, Vieill.

(E). MAMMALS MOUNTED SINGLY UPON STANDS AND MAMMAL HEADS.

476 Northern Hare. Le Lièvre du Nord.

Lepus americanus virginianus, Harlan.

477 Musk Rat. L'Ondatra Musqué.

Fiber zibethicus, I.

478 Skunk. Le Zorille Varie.

Mephitis mephitica, Shaw .

479 Mink. Le Vison d'Amerique.

Putorius rison, Schreber. 
480 Red Deer Heads. Le Cerf de Virginie.

Cariacus virginianus, Boddaert.

481 Moose Heads. L'Elan d'Amerique.

Alce alces americanus, Jardine.

482 Woodland Caribou or Reindeer Heads. Le Renne Caribou.

Rangifer tarandus caribu, Kerr.

\section{OOLLEOTION OF BIRDS; MOUNTED IN CASES.}

922 Col. Thos. J. Egan, Halifax, Nova Scotia.

CASE A.

Common Loon or Great Northern Diver. Le Plongeon a Collier.

483 Male.

Urinator imber, Gunner.

484 Female.

485 Two young ones.

Reü-throated Diver. Le Plongeon du Nord.

486 Male.

Urinator lumme, Gunner.

487 Female.

Red-necked Grebe. Le Grebe a Cou Rouge.

488 Male.

Colymbus holboellii, Reinhardt.

489 Female.

Razor-billed Auk. Le Pingouin Commun.

490 Two Males.

Alca torda, Linnæus.

491 Female.

Puffin. Le Macareux Arctique.

492 Male.

Fratercula arctica, Linnæus.

493 Female.

Black Guillemot. Le Guillemot Noir.

494 Male.

Cepphus grylle, Linnæus.

495 Female.

496 Young. 
CAST: B.

White (iannet or solan fiogse. Te fion de Bassan, on Murgmen. 497 Malc.

498 Female.

49.) Bungomaster Ginll. Le Goeland it des Blenatre. Larusglaucus, Brumich.

5(i) White-winged (iull. Le Goelund it Ailes Blamches.
lacus leucople'rus, Faber.

501 Richardison's Jaeger. Le stercoraire Areticue.

Stevcorarius parasiticus, liuniens.

Aretic or Long-tailed Jateger. Le Stercoraire de Buffom. 502 Two Males.

Stercorarius longicaudus, Vieillot.
les.

503 Female.

\section{('ASE C.}

Inoble crested Cormorant. Le Cormoran a Aigrettes. 504 Male. Phalacrocorax dilophus, Swainson and Richardson.
505 Fenale.

Wandering Shearwater. Le Girand Puftin.

506 Male.

Puffinus major, Faber.

507 Female.

5 (k Aretic Jaeger. Le Stercoraire de Buffon.

Stercorarius longicandus, Vieillot.

Dusky Shearwater. Le Puflin Noir.

509 Male.

Puffinus anduboni, Finch.

510 Female.

Fulmar. Le Petrel Glacial.

5 tl Male. Finlmarus glacialis, limnens.

512 Female.

Yellow-billed Tropic Bird. Le Phaton.

513 specimen of male. 
CASF: 1).

514 Burgommater ciull. Le cioelund it don Blenutre. Jarus glaucus. Brmunich.

Imericun Herring Gull. Io Cioelnud A rgenté.

515 Male.

Lames urgentatus imitheomiawes, Cones.

516 fiemule.

517 liggen.

Langling Gull. La Monette Riense.

518 Male.

Larus atricilla, Linnaeus.

519 Female.

5E) Bunaparte's Giull. Le Goelond de Bonapurte.

l.arus philadelphia, Ord.

Murie. Lo Guillemot i Ventre Blanc.

Uria troile, Linnaeus.

521 Male in Summer.

522 Femaic in Winter.

Dokekie or Little Auk. Le Mergule Pigeon.

Alle alle. Linnaeus.

523 Male.

524 Female.

525 Dabchick or Little (irebe. Ite (irebe a Bec Bigarre.

Podilymbue podiceps, Liunaeus.

Horned (irebe. Le Grebe Cornu.

526 Male.

.727 Female.

\section{CASE is.}

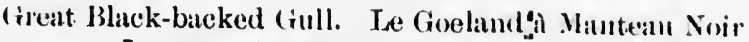

$52 s$ Male.

Larus marinu:, Linnaeus.

529 Female.

530 Eggs.

Kittiwake (iull. La Mouette a 'Trois Inoigts.

j31 Male.

Jarus tridactyln, Limmeus.

532 Female. 
Ivory (inll. La Mouette Blanche.

593 Male.

Gurion allor, (iunner.

534 Femule.

Canpian Teru. La surno Canpiemuc.

Stirna tachegram, Idpech.

535 specimen of male.

Roseate Tern. La Sterne Romere.

Sterna dougalli, Montague.

636 specimen of male.

537 Common Tern. La sterne de Wilsun.

stern" hirundo, linnacus.

Sis Aretic 'Tern. La Sterne Aretique.

Stirma poradisaen, Brmnn.

Black Tern. La Sterne Noire.

539 Young male.

Hydrochelidom nigra surinum.nsis, Gincliu.
male.

540 Young female.

CASE $F$.

Canada Goose. La Bermache du C'anada.

inl Npecimen of male.

Brunta canudensis, linnaeus.

Brant Goose. La Bernache Commmut.

oft2 Male.

Branta berniclı, linnaens.

543 Female (albino).

American Merganser. Le Harle d'Amerifune.

544 Male.

Mergunser americanus, Cass.

545 Female (a) and young specimens (b).

Hooded Merganser. Le Petit Harle.

546 Male.

Lophodytes cucullotus. I.innarus.

547 Female. 
Dusky Duek. le Canard Noir. Anas obscura, Gmelin.

548 Male.

549 Female (a) and young specimens. (b)

Mallard. Le Canard Ordinaire. 550 specimen of Male. Anas boschas, Ifimneus.

shoveller. Le Canard Souchet.

551 ivale. spatula clypersa, Linnacus.

552 Female.

American Widgeon. Le Canard Sifteur d'Amerique.

553 Nale. Anas americamus, Gmelin.

554 Female.

Wood Duek. Le Canard Huppé

555 Male.

Aix sponst, Limners.

556 Female.

Blue-winged Teal. La Sarcelle ì Ailes blinurs.

$55 \%$ Male.

Anas discors, Linnarus.

558 Female.

(ircen-winged 'Teal. La Sarcelle ì Ailes Vertes. Anas carolinensis, Gmelin.

j59 Male.

5 (60) Fenale.

('ASL H.

Wider luek. L' Eider Commnn.

5 (il Male.

562 Feinale.

56.3 Eggs.

564 Barrow's Golden bye. Ie Bucéphale d'Islande.

Glancionetta islandica, Gmelin.

itis) Anerican (iolden-liye. Le Bucéplale d'Anericue.

cilaucionetta cirngulir americana, Butaparte. 
Isulg-tailed Duck. Le Canard a Longue Qume. 566 Male in summer. Clangula hyemalis, Linnoris.

567 Male in winter.

568 Female specimen. 569 American Scaup Duck. Ie Morillon à tête Noire.
Avthya marila nearctica, Stejneger.

570 Buftle-head. Le Petit Bucéphale, Charitonetta albeola, Iinnitus.

\section{('ASE: I.}

King Eider. I'Eider Remarquable.

571 Male. Somateria spectahilis, Limmans.

572 Female.

A merican scalup Duck. Le Morillon à tete Noire.

57: Specimen of Male.
Alla neartica, Stejneger.

Harleguin Duck. Le Canard Histrion.

574 Male. Histrinnicus histrionicue, I.imnerus.

575 vemale.

White-winged scoter. La Macreuse Velontr.

576 Male.

Oidemia deglandi, Bomaparte

577 Female.

Nurf Scoter. Ite Canard Macreuse à lauge Bece.

578 specimen of Male.

Oidemia perspicillata. Tinnsels.

American scoter. Le Canard Macreuse d'Amerique.

579 Specimen of Male.

Red-breasted Merganser. Ite Harle à Poitrine Ronsese.

580 Male.

Merganerer scrrator, Timmeus.

581 Female. 
CASE J.

Great Blue Heron. Le Grand Héron Blell. Arden herodias. Iinnieus.

582 Male.

583 Female.

584 Young specimens and eggs.

Anerican Coot. La Fonlque d'Amerique.

585 Male.

Fulica americana, Gmelin.

586 Female.

American Woodcock. Le Bécasse d'A merique.

587 Male.

Philohela minor, Gmelin.

588 Female.

589 Nest and eggss.

Wilson's Snipe. La Bécassine de Wilson.

590 Male.

Gallinago delicata, Ord.

591 Female.

592 Young.

CASL: K.

Gireat Blue Heroı. Le Girand Héron Bleı. Ardea herodias, Linureus.

593 Specimen in young plumage.

American Bittern. Le Butor.

594 Male.

Botaurus lentiginosus, Mont:ig.

595 Female.

596 Specimen of young.

Green Heron. Le Héron Vert.

597 Specimen of Male.

Arden virescens, Linnmus.

Least Bitteru. Ie Petit Butor.

Botaurus exilis, Gmelin.

598 specimen of Male.

Virginia Rail. Le Rale de Virginie.

Rallus virginianus, linn:eus.

5 g9 Specimen of Male. 
Common Rail or Sora. Le Rale de la Cimoline.

$\$ 00$ Male.

Porzana porzana, Linuaeus.

601 Female.

Lenst or Black Rail. Le Rale Noir.

602 Spetimen of male.

('ommon Gallinule. La Gallinule de la Floride.

to3 Male. G'ollinula galeata. Licht.

tor Female.

Hudsonian Curlew. Le Courlis de la Baie d'Hudson.
Male. Iumenius inudsonicus, Lath.

605 Male.

606 Femali.

\section{CASE I.}

Brown Pelican. Le Pélican Brun.

(iot specimen of Male.

American cireat White Lgret. Le Heron Bianc. wis specimen of Male. Arden egrita, fintelin. Le Héron Blame.
of Male.

Black-cirowned Night Heron.

(6)9 Male.

Niycticorax nuteron. Le Héron du Nuit.

(i) Yoming Finule.

King Rail. Le Rale Élégant.

611 Male.

Rallus slcqans, Avdubou.

112 Female.

Little Bhe Heron. Le Petit Héron Blen.

013 sperimen of male.

lapwing. La Vameau Huppé.

Vanellus cristatus. 
( ASE M .

Great Marbled Godwit. La Barge Marbrin.

(615 Male.

$$
\text { Limosa fedoa, Limnaus. }
$$

ti16 Female.

Hudsonian (rodwit. La Barge de la Baie d'Hudson.

Limosa har mastica, Liunzens.

(i17 Male.

(618 Female.

Willet. Le Chevalier Semipalmi.

619 Male.

Sumphemia semipalmata, (imelin.

620 Fenale.

American Golden Plover. Le Pluvier l)ore.

1 Charadrius dominirus, Müller.

(i21 specimen of Female.

Black-bellied Plover. Le Siquatarole ì Ventre Noir.

Charadrius squatarolu, limmens.

ti22 Male.

i23 Female.

(ireater Yellow Lege. Le Chevalier Aboveur.

Totanus melanoleucus, rimelin.

(624 Male.

ti25 Female.

Lessere Yellow Slanks. Le Petit Chevalier à pieds Jaunes.

Totanus flazipes, Gmetin.

126 Male.

627 Female.

Piping Plover. Le Pluvier Criard.

Agralitis meloda, Ord.

ii28 spreeimen of Male.

Kildeer Plover. Le Pluvien Kildir.

Agialitis rocifera, linnans.

fizy specimen of Male.

Ring-neck Plover. Le Pluvier Semipalme.

630 Male.

. Egualitis semipalmata, Bomaparte.

ti3! female, 
Spotted Sand-piper. La Maubeche Tachetive.

632 Mule.

Actitis macularia, Ioinnacus.

633 Female.

634 specimens of yourng.

Solitary Sand-piper. Le Chevalier solitaire.

635 Male.

Totanus soliturius, Wilson.

636 Female.

sanderling. Le sanderling.

637 Male.

Calidris urenuria, Limnaens.

638 Female.

Teasst Nand-piper. La Maubíche de Wilson. t39 Sprecimen of Male.

Tringa mimutilla, Vieillot.

Pectoml Sand-piper. La Maubéche Tachetée.

t540 Male.

Tring" maculatu, Vieillot.

fi41 Female.

Buff-breasted Sand-piper. La Maubeche ì P'oitrine jaunatre.

t542 Male.

Trymgites sulirufirollis, Vieillot.

fi43 Female.

Long-legged Sand-piper. L'Echasse à Long Pieds.

lit4 Male.

Micropalami himantopus, Bonaparte.

1455 Female.

Kunt. "I' Ash-eolored Sami-piper. La Maubéche a Poitrine

646 Male.

Tringa cautus, linnaens. Rousse.

i47 Female.

P'urple Sand-piper. La Maubéche Pourpre.

ti48 Male.

Trtnga maritima, Brunn.

649 Female. 
Turnstone. Le Tournepierre at Poitrine Ninire.

(i50) Male.

Arenaria interpres, I.innaens.

651 Female.

652 Young male.

Wilson's Phalarope. Le Plaalarope de Wilson.

653 Male.

Phalaropus tricolor, Vieillot.

654 Female.

Red Phalarope. Le Phalarope Roux.

655 Male.

Crymophilus fulicarius, Linnaens.

656 Female.

Northern Phalarope. Le Plaalarope Hyperbircen.

657 Male.

Phaluropus lobatus, linnaens.

658 Female.

659 Male in winter plumage.

( ASE N.

Canada Grouse. Le Tétras dı Canada.

660 Male.

Dendragupus cenadr"sis, linnaw:ıs.

661 Female.

Canadian Ruffed Grouse. La ciélinutte à liraim.

662 Male.

Bonasa umbellus togmta. Iinnaells.

663 Female.

664 Specimens of young.

Dusky Girouse.

665 Male.

Demlragamus olsscurus, siḷ.

666 Femalı.

Willow Grouse. Le Lagopède des sinules.

667 Male.

lagopus lugopus, I,inmaens.

668 Female.

Rock Ptarmigan. Le Lagopide des Richlers. 669 Specimen of Male.

Lagopus rupestris, (imelin. 
43

Sharp-tailed (irouse. Le Gélinotte à quene Aigue .

670 Male.

Prdincirtex phasianellus, I,innacus.

(B71 Female.

Pinnated Gronse or Prairie Hen. La Gélinotte des Prairies. 672 Male.

Tympanuchus americanus, Reich.

673 Female.

Virginia Quail or "Bob White." La raille.

674 Male.

Colinus virginianus, Linnens.

675 Two specimens of Female.

\section{CASE 0.}

676 Willow Grouse. Le Lagopède des Sanles.

Lagopus lagopus, Linnaeus.

Specimens exhibiting all the seasonal changes of plumage.

('ASE P.

677 Bald liagle. L'Aigle a tete Blanche.

Haliztus leucocephalus, Limiaeus.

A pair of mature birds with their young.

CASE Q.

678 (io!den Eagle. L'Aigle du Canada.

Aquila chrysaetos, Tinnaeus.

Marslı Harrier. Le Busard des Marais.

679 Male.

Circus hudsonius, Linneus.

680 Female.

Nharp-slıinned Hawk. L'Epervier Bruı.

681 Male.

Accipiter relox, Wilson.

682 Female.

ti83 Specimens of young. 
American Sparrow Hawk. Le Fancon bipervier .

684 Male.

Fuleo spurverius, Iinuens.

685 Female.

Pigewn Hawk. Le fiancon des Pigeons.

686 Male.

$$
\text { Filio columlarins, Limniris. }
$$

(387 Female.

(i88 Nest and Eggs.

American Sparrow Hawk. Le Eaucon Hpervier.

ti89 speciume Falco Sparverius, Liminss.

690 'Turkey Vulture, Le Vautour Aura.

Cithurtes aura, Iinnaens.

$$
1 \text { CASE R. }
$$

American Osprey or Fish Hawk. Le Balbusard de la Caroline

691 Male.

Pandion haliaetus curolinensis, Gmelin.

692 Female.

693 Young Speciutens.

lnck Hawk. Le Fancon Pélerin.

694 Male.

Halco peregrinus anatum, Bonapat'i.

695 Female.

Redl-tailed Hawk. La Buse à Qnene Ronsso. Briteo borealis, Gmelin.

$69 t$ A specimen in first plimage.

\section{'CASE 8.}

Auerican Goshawk. L'Autour î téte Noir.

697 Male.

Acripiter atricapillue, Wilson.

698 Femiale.

699 Specimens of young.

Red-tailed Hawk. La Buse à Quene Ronsst.

700 Male.

Butro lonecolis, Gullelin.

701 Female.

702 Specimens of young. 
Cima (iyr-falcous. Le: Fatucon Noir. 703 sipecimen of mals.

falco musticolus, Liunaen.

IASE T.

American rungh-legged Buzzard. La Buse L'attue d'Amerique.

704 Male.

Archibuteo lagopus suncli-johannis, Cinelin.

705 Fenale in first plumage.

Broad-winged Hawk. La Buse de Pemsylyanie. Tori specinen of malc.

Buteo latissimus, Wilson.

American Hawk Uwl. La Chouette Eperviere d'A merieque.

707 Male.

Surnia ululu cuparoch, Muller.

708 Female.

Great ciray ()wl. La chouette cendrée.

ins sipecinem of Male.

Ulula cinerea, timolin.

710 Western Horned Owl. Le Dnc de Virginie.

Bubo rirginiunus subarcticus, Hoy.

Sharp-shinmed Hawk. L'Epervier Brun. Accipiter zelo.t, Wilson.

711 specimen in young pluniage.

\section{CASE $\mathrm{U}$.}

Cireat Horned Owl. Le Duc de Virginie.

712 Male.

Bubo ariginianus, Gmelin.

713 Feinale.

714 specimens of young.

Barred Owl. La Clivuette du Cillada.

715 Male.

Syrnimm nehnlosum. Forster.

716 Female. 
Snowy Owl. Le Ilarfung.

717 Male.

Nutea nyctea, Limurus.

718 Fomale.

Short-eared Uwl. Le Hibou a digrelte (inturtes.

719 Male.

Asio accipitrinus, Pallas.

720 Female.

American I ong-eared Owl. L, Hibon a Aigretien Langues.

721 Male.

Asio avilsonianus, Jesison.

722 Female.

Acadian Owl. Ia Nyctale d'Acadic.

723 Male.

Nyctala acadia, (imelin.

724 Feniale.

Richardson's Owl. La Nyctale de Richardson.

725 Male.

Nyctala ten.rmalmi richardsoni, Bonaparte.

726 Female.

\section{(ASE V.}

Northern Raven. Le Corbeau dı Nord.

727 specimen of male.

Cormus corax principalis, Ridgwiy.
en of male.

American Crow. La Corneille d'Amérique.

728 Male.

Corrus americanus, Audubon.

729 Female.

Red-winged Blackbird. L'Fitourneau ì Ailes Rough.s.

730 Male.

Agelaius phoeniceus, Iinnieus.

731 Female.

732 Specimen of youmg male.

Rusty Grackle or Thrush Biackbird. Ia Mainate Couleur de

733 Male.

Scolecophagus carolinus, Miiller. Reruille.

734 Female. 
Bronzed dirackle or Crow Blackbird. Ice Mainate Bronzi.

Belted Kingtislier. Le Martin Picleenr.

736 Male.

Cirrole alcroun, Jinureus.

737 Fentale.

738 Albino sprecinen.

Ilairy Woodpe aker. Le Pic Clieveli.

739 speecimen of males villosus lencomelas, Budel.

Lowny Woodpecker, le Pic Mimnle.

740 Male.

mryohutes puhescens, Limmans.

741 Fernale.

Aretic Three-toed Woudpecker. Ie Pie Areticyue.

74t Male.

Picoides arcticus, swainson.

743 Female.

Yellow-bellied sap-suckel. Le Pic Maenle.

$7+4$ specimen of Male.

Pileaterl Woodpecker. Le Pic a Huppé Ficarlate.

745 Two specimens.

Cophloeus pileatus, linnseus.
cimens.

Red-lieaded Wootpecker. If: Pic à tîte Rouge.

itti specimen of male

$$
\begin{aligned}
& \text { Melanerpes aryhlirocephalus, Linnals. } \\
& n \text { of male }
\end{aligned}
$$

Ciolelen-winged Woodpecker or Flicker. Ie Pic Dorí.
Male. Colaptes auratus. Tinnmus.

747 Male.

748 Female.

Night llawk. L'Engoulevent d'Amírique.

749) Male.

Chordeile's airgivianus, Gmelin.

50 Specinten of young. 


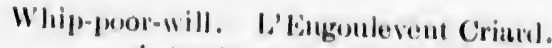

int specintert of Mule.

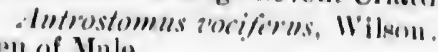

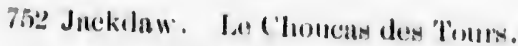

Cormes momedulu.

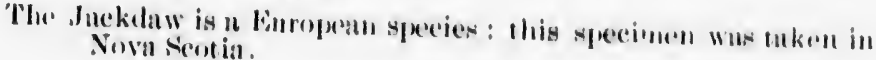

C.ISI: II.

Paswenger l'igeon.. la l'igenn Vungugent.

753 Male.

lictopiste's migrutorius, limmens.

754 Female.

Manruing or Carolina Dove. La Tourterefle de ha Caroline.

755 Male.

Zenuidilra marontu, Limurens.

75 ti Female.

Yollow-bellied Ćneken. Le Concond'Anérique.

Coccyzus americum!s, Limnems.

757 specimen of Mala.

Black-billed Cnekou. Le Concun it Bec Nuir.

75s Male.

Coccyous erythrophthalmus, Wilion.

759 Female.

760 Nest and egges.

King-bird or Bee Martiu. Le Tyran de la caroline.

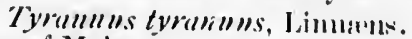

761 specino'n of Male.

Olive-sided Flycatcher. La Mencherolle à (ôtes ()live.

T62 Male.

Contopus boralis, Swaiuson.

763 Female.

Wood Pewee. Ie Moncherolle Verdatie.

764 Male.

Contopus zirens, Iimnins.

765 Female. 
Shore or Horned Iark. L'Alenetle ordimite on de Virginie. 766 Male. Otocoris alpestris, Limmens.

767 Female.

Meadow Lark. L'bton'nenu des l'ris.
Sturnella magrua, Linuens. 768 Specimen of Fenale.

Purple Fincl. Le Pinson Ponrpro,

769 Male.

Carpodacus purpureus, Gundiu.

770 Female.

771 Nest and eggs.

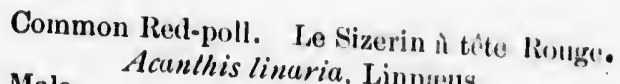
772 Male.

773 Female.

American Gold-finch. Ite Chardonneret Jaune.

774 Male.

Spinus tristis, Linneus.

775 Female.

Pine Siskin. Le Chardonneret des]Pins.

776 Male.

Spinus pinus, Wilson.

777 Female.

Snow Bunting. Le Plectrophane deg Neiges.

778 Male.

Plectrophenax nivalis, Linniens.

779 Female.

Savanna Sparrow. Pe Pinson de Savanna.

7:0 Male.

Ammodramus princeps, Mayn.

781 Female.

White-throated Sparrow. Le Pinson a Gorge Blanche.

782 Male.

Zonotrishia albicollis, Gmelin.

783 Female.

784 Nest and eggs 
Tree Sparrow. Le Pinson de Montigne.

785 Male in winter.

786 Female in suminer.

Eastern Snow-bird. Le Pinson Niverolle. Junco hyemalis, Linn:eus.

787 Female with nest and eggs.

Song Sparrow. Le Pinson Chanteur.

788 Male. Melospiza fasciata, Gmelin.

789 Female.

790 Nest and eggs.

Swamp Sparrow. Le Pinson de Marais.

791 Male.

Melospiza gev, yiana, Iath.

792 Female.

Fox Sparrow. Le Pinson Fauve.

793 Male.

Passerella iliac", Merr.

794 Female.

Red-eyed Vireo. Le Vireo aux Yeux Ronges.

795 Male.

Vireo olivaceus, Linnieus.

796 Female.

Warbling Vireo. Le Vireo Gris.

Vireo gilvus, Vieillot.

797 Specimen of male.

Solitary Vireo. Le Vireo â Tête Bleuâtre.

798 Male.

Virio solitarius, Wilson.

799 Female.

Black and White Warbler. Le Grimpereau Noir et Blanc. 800 Male.

Mniotilta varia, Linneus.

801 Female. 
802 Male.

Blue-yellow-backed Warbler. La Fauvette d'Amerique.

803 Female.

Cape May Warbler. La Fauvette du Cap May

804 Male.

Dendroica tigrina, Gmelin.

805 Female.

Yellow Warbler. La Fauvette Jaune.

806 Male.

Dendroica restiva, Gmelin.

807 Female.

808 Nest.

Black-throated Blue Warbler. La Fanvette Blen ì Gorge Noire. 809 Specimen of male.

Dendroica caerulescens, Gmelin.

Yellow-rumped Warbler. La Fauvette ì Croupion Jaune.

810 Male.

Dendroica coronala

811 Female.

Magnolia Warbler. La Fauvette ì tête Cendrée.

812 Specimen of male.

Dendroica muculosa, Gmelin.

Chestnut-sided Warbler. La Fauvette de Pensylvanie.

813 Male.

Dendroica pensylvanica, Linnieus.

814 Female.

Bay-breasted Warbler. La Fauvette ì Poitrine Baie.

815 Male.

Dendroica castanea, Wilson.

816 Female.

Black-poll Warbler. Ia Fauvette Rayée.

817 Male.

Dendroica striata, Forster.

818 Female.

Blackburnian Warbler. La Fauvette de Blackburn.

819 Male.

Dendroica blacklinurit', (intelin.

820 Female. 
Black-throated Green Warbler. La Fauvette à Poitrine Noire. 821 Male. Dendroica virens, (imeliı.

822 Female.

Yellow Red-poll or Palm Warbler. La Fauvette a tête Rousse. 823 ' Male. Dendroica palmarum hypochrysea, Ridgway.

824 Female.

Nashville Warbler. La Fauvette de Nashville.

825 Male.

Helminthophila ruficapilla, Wilson.

826 Female.

Maryland Yellow-throat. La Fauvette Trichas.

827 Male.

Geothlypis trichas, Linnwus.

828 Female.

American Redstart. Ia Fauvecte Moucherolle Dorée.

829 Male. Setophaga ruticilla, Linnieus.

830 Female.

831 Nest and eggs.

Canadian Fly-catching Warbler. Ia Fauvette Moucherolle du

832 Male.

Sylvania canadensis, Limmeus.

Canada.

833 Female.

Chipping Sparrow. Le Petit Pinson à Couromme Rousse. Spizella socialis, Wilson.

834 specimen of male.

\section{CASE $\mathbf{X}$.}

Ruby-throated Humming Bird. Ie Colibra ì Gorge Rubis. Trochilus colubris, Linnaus.

835 Male.

836 Feinale.

837 Nest and eggs.

Blue Jay. Ie Geai IIupux́.

838 Male.

Cyanocilla crislata, Limmous.

839 Fenale. 

Cat-bird. La Grive de la Caroline.

861 Male. Galcoscoptes corolinensis, Linneus.

862 Female.

Winter Wren. Le Troglodyte d'Hiver. 863 Specimen of Male. Troglodytes hiemalis, Vieillot.

Brown Creeper. Le Grimperenu d'Amérique.

864 Male. Certhia familiaris americana, Bonaparte.

865 Female.

White-breasted Nuthatch. La Sittelle de la Caroline. 866 Specimen of male.

Sitta carolinensis, Lath.

Red-breasted Nuthatch. La Sittelle du Canada. 867 Specimen of inale.

Sitta canadensis, Linnreus.

Hudsonian Chickadee. La Mésange de la Baie d'Hudson.

868 Male

Parus hudsonicus, Forster.

869 Female.

Black-capped Chickadee. La Mésange à tête Noire 870 Specimen of male.

Parus atricapillus, Linneus.

Golden-crowned Kinglet. Le Roitelet Huppé.

871 Male.

Regulus satrapa, Licht.

872 Female.

Ruby-Crowned Kinglet. Le Roitelet ì Couronne Rubis.

873 Male.

Regulus culendula, Lim1eus.

874 Female.

Water-thrush. Ia Grive Hochequene.

875 Specimen of male.

Seiurus noreboracensis, (imelin. 

Golden-crowned Thrush or Over: Bird. La Grive Couronnée.
Seiurus aurocapillus, Linneus. 876 Male.

877 Female.

878 Nest and eggs.

Wilson's Thrush. La Grive de IVilson.

879 Male.

Turás fuscescens, Stepl.

880 Female.

Olive-backed Thrush. La Grive de Swainson.

881 Male.

Turdus ustulatus swainsonii, Cab.

882 Female.

883 Nest and eggs.

Hermit Thrush, La Grive Solitaire.

884 Male.

Turdus aonalaschkae pallasii, Cab.

885 Female.

886 Nest and eggs.

Americain Robin. Le Merle d'Amérique.

887 Male.

Merula migratoria, Linnieus.

888 Female.

Brown Thrasher. La Grive Rousse.

889 Specimen of male. 
923 Dominion of Canada, Department of Marine and Fisheries. Sir Louis H. Davies, K.C.M.G., Minister. Lieut.-Col. Gourdeau, Deputy Minister. Professor Andrew Halkett, Naturalist.

Mounted Fishes and Crustaceans.

Marine Invertebrates, small fishes, etc., arranged in case in a series of trays.

Miscelloneous Specimens, embracing Shells of Lamellibranches, Echinoderms, etc.

Models of Canoes, and Fishing Gears.

\section{MOUNTED PISHES AND CRUSTACEANS.}

890 California Dog-Fish. L'A iguillat de Californie.

Squalus sucklii, Girard

Female From British Columbia.

2. Mounted on Standards.

891 Picked Dog-Fish. L'Aiguillat Commun.

Squalus acanthias, Linnsus.

Specimen from Gulf of St. Lawrence.

892 Porbeagle or Mackerel Shark. - La Lamie.

Lamna cornubica, Gmelin.

Female from Grand Manan, New-Brunswick ; also Foetus of Shark from the same locality.

\section{Loaned by Mr. Henry Gatehouse, of Montreal.}

893 Male from Gulf of St. Lawrence.

$89+$ Fenale from Gulf of St. Lawrence.

895 Barn-door Skate. La Raie.

Raja latevis, Mitcliill.

Female from New Brunswick.

896 ( ) Rat-Fish or Elephant-fisl. La Chimère de Collie.

Chimiera colliei, Bennett.

Male, female, and egg-capsule from Coast of Britisl Columbia.

(B) Bowfin or Dog-fish. Poisson de Vase,

Amia calva, Linnacus.

'Two specimens from Lake Ontario. 
897 Common Gar-pike. Le Lepidostce Osseux.

Lepidosteus osseus, Limnaeus.

Male from Deschene Lake, Province of Qnebec.

$$
\text { Mounted on Standaidis. }
$$

898 (A) Common Gar-pike. Le Lepidostée Osseux.

$$
\text { Lepidosteus osseus, Limnaeus. }
$$

Specimen from Ottawa River.

(B) Lake Sturgeon. L'Esturgeon des Lacs.

Acipenser rubicundus, LeSueur.

Specimen from Lake Ontario.

899 Common Sturgeon. L'Esturgeon Commun.

Acipenser sturio oxyrhynchus, Mitchill.

Seven specimens from Lake Erie.

900 A specimen some eight feet long, from Gulf of St. Lawrence.

901 White Sturgeon. L'Esturgeon Blanc.

Acipenser transmontanus, Riclardson.

Specimen from month of Fraser River, British Columbia. 150
lbs. weiglit.

902 Paddle Fisl. L'Esturgeon Spatulaire.

Polyodon spathula, Walbaum.

Specimen from Lake Huron, near Sarnia, Ontario.

903 (A) Common Cat-fish or Bull-head. Ia Barbotte.

Ameinrus nebulosus, Le Sueur.

Two specimens from Lake Ontario.

(B) Great Fork-tailed or Mississippi Cat. La Barbue.

Ameinrus lacustris, Walbaum.

Specimen from Lake Ontario.

901 Great Fork-tailed or Mississippi Cat. La Barbue.

Ameiurus lacustris, Walbaum.

umbia.

Two specimens from Red River, Manitoba.

905 Buffalo-Fish. Le Buffle Poisson.

Ictiobus bubalus, Rafinesque.

Three specimens from Lake Winnipeg. 
906 (A) Suall-scaled Sucker. Ie Cyprin Commersonnien.

Catostomus commersonii, Laeépide.

Two specimens from Lake Ontario.

(B) Large-scaled Sucker. Ie Moxostîne Doré.

Moxostoma aureolum, Le Sueur.

Specimen from Lake Ontario.

907 Large-scaled Sucker. La Moxostôme Doré.

Moxostoma aureolum, Le Sueur.

Two specimens from Lake Winnipeg, North West Territories.

908 Moon-eye or Toothed Herring. L'Hyedon Terzisse.

Hiodon torgisus, Le suenr.

Five specimens from Lake Ontario.

909 Common Herring. Le Hareng Commun.

Clupea harengus, Linnaens.

Specimens from Magdalen Islands.

910 American Shad. I'Alose d'Amerique.

Clupea sapidissima, Wilson.

A specimen from River St. Lawrence.

911 American Smelt. L'Eperlan d'Amerique.

Osmerus mordax, Mitchill.

Eight specimens from Miramichi River, New Brunswick.

912 Common White Fish. Le Poisson Blanc.

Coregonus clupeiformis, Mitchill.

(A), (D), (E), 3 small specimens from :ake Simcoe, Ontario.

(B), (C), 2 large specimens from North West Territories.

913 Cisco or Lake Herring. Le Scisco.

Corcgonus artedi, Le Sueur.

Six specimens from Lake Frie.

914 Quinnat or Clinook Salmou. Le Quinnat.

Oncorhynchus tschazytscha, Walbaum.

Specimen from British Columbia.

915 Quinnat or Chinook Salmon. Le Quinnat.

Oncorhynchus tschawytscha, Walbaum.

Five small specimens, reared by Samuel Wilmot, Esq., Fish Culturist, in his garden, Newcastle, Ont; 4 years old; eggs
from California. 
916 (A) Blue-back Salmon, or Suck-eye. Le Nerka.

Specimen forhynchus nerka, Walbaum.

(B) Dog-salmou, Le Keta.

Oncorinynchus keta, Walbaum.

Two specimens from Coast of British Columbia.

917 (A) Ouananiche. L'Huananicle.

Salmo salar ouananiche, McCarthy.

Specimen from Lake St. Jolın, head of Saguenay River, I'rovince

(B) Atlantic salmon. Le saumon comnun.

Salmo salar, Linnaeus.

Specimen from Restigouche River.

918 Atlantic salmon. Le Saunon Commun.

Salmo salar, Limmaeus.

Two specimens from Lake Ontario.

919 Salmon Trout, or Great Lake Trout. La Truite des Laes.

(A) Specimelinus namaycush, Bloch.

Superior, Ontario.

920 speckled or Brook Trout. Le Truite des Ruisseaux ou Tachetée

Salvelinus fontinalis, Mitchill.

(A) Two specimens from Rock Lake, Haliburton County, Ontario.

From Mr. Andrew Fleck, of Parry Sound Railway.

Ontario.

ritories.

Fish Cul-

(B) A specimen from Nepigon Bay, Lake Superior, Ontario.

921 Speckled or Brook Trout. La Truite des Ruisseaux ou Tachetéc

Salzelinus fontinalis, Mitchill.

(A) Two specimens from German Lake, Province of Quebec.

(B) A specimen from Restigouche River.

922 Red-spotted or Dolly Varden Trout. La Truite " Dolly Varden."

Salvelinus malma, Walbaum.

Two specimens from Coquitlam River, a tributary of Fraser

923 Green Pike. Le Brochet hêtieulé.

Esox reticulatus, Le Sueur.

Four specimens from Lake Memplremagog, Province of Quebec. 
924 Common Pike. Le Brochet Commun.

Esox lncius, Linnaeus.

Two specimens from North West Territories.

925 (A) Common Pike. I, Brochet Commun.

Esor lucius, Limnaens.

Specimen from Lake Ontario. Also a very smnll specimen from same locality.

(B) Musketlunge. Le Maskinongé.

Eso.x nobilior, Thompson.

Specimen from Bay of Quinte, Ontario, 35 tbs weight.

926 Muskellunge. Le Maskinongé.

Esox nobilior, Thompson.

(A) Specimen from Bay of Quinte.

(B) Specimen from Rideau River, 16 Hbs. weiglit.

927 American Eel. L'Anguille d'Amerique.

Angnilla anguilla rostrata, Le Sueur.

Specimen irom Rideau River, Ontario. Collected by Mr. J. M. lalonde, Department of Marine and Fisheries.

928 (A), (C), Common Mackerel. Le Maquereau Commun.

Scomber scombrus, Linnaeus.

Four specimens from Gulf of St. Lawrence.

(B), Spanish Mackerel. Ie Tassard Tacheté.

Scomberomorus maculatns, Mitchill.

Two specimens from Gulf of St. Lawrence.

929 Tunny or Horse Mackeret. Ta Thonine, ou Touna. Ie Thon.

Thynnus thynnus, Linnaens.

Specimen from Sagnenay District.

Mlounteci on Standards.

Weiglit some $400 \mathrm{lbs}$.

930 Two swords of Sword-fish. L'Wspadon.

Xiphias gladius, Linnacus.

From Atlantic coast of Canada..

931 (A) Calico Biass or Strawberry Bass. Le Crapet Calicot.

Pomoxys sparoides, Lacépède.

Two specimens from Burlington Bay, Ontario. 
(B) Striped Basy. Le Bar Anericain.

Roccus lineatus, Bhoch.

Five specimens from Miramichi River, Now Brunswick.

(C) Whito Bass. Le Bar Blanc.

Roccus chrysops, Rafinesculu.

Two specimens from Lake Erie, Ontario.

932 Striped Bass. Le Bar Américaill.

Roccus lineatus, Bloch.

Two specimens froul Miranichi River, New Brunswiek.

933 (A), (B), (E), (F), Rock Bass. Le Crapet Vert.

Ambloplites rupestris, Ratinesque.

Four specimens from Lake Ontario.

(C), (D), Sun Fish. I Crapet Jaune.

Lepomis gibbosus, Linnaeus.

Two sperimens from Lake Ontario.

934 (A), Large monthed Black Bass. I'Achigan Grande Bouche. Micropterus salmoides, Lacépìde.

A specimen from Lake Erie, Ontario.

(B) Small mouthed Black Bass. L'Achigan Petite Botche. Micropterus dolomicu, Lacépìde.

A specimen from Rice Lake, Ontario.

935 Small-mouthed Black Bass. L'Achigan Petite Bonche.

Micropterus dolomieu, Lacépède.

Five specimens from Bay of Quinte.

936 Yellow Perch. La Purche.

Perca facescens, Mitchill.

Five specimens from Bay of Quinte.

937 Pike-perch. Le Grand Dorè.

Stizostedion vitreum, Mitchill.

(A) Two specinens from Rideau Lake.

(B) A specimen from Lake Huron.

938 Sheepshend or Fresh water Drun. Le Malachigan.

Aplodinotus grunniens, Rafinesque.

(A) A specimen from Detroit River, Ontario.

(B) A specimen from Ottawa River. 
039 (A) Rock-trout or Boregnt. Le Boregnt.

Heragrammos decagramumus, Pnllas.

Specimen from Esquimalt Inrbour, Britisl Columbia.

(B), (C), Cultus Cod. Ia Morne Cultus.

Ophiodon elongralus, Girard.

Two specimens from Victoria, Vuncouver Islnud.

Obtained in deep water.

940 Sinapner. Le Sébaste.

Sebastes mariuns, limmeus.

A splecimeu from Atlantic coast of Canada.

941 Red Rock-flsh. Le Tambor.

Sebaslode's ruberrimus, Cramel.

A specimen from Coast of British Columbia.

942 (A) Lump-fish. Le Cyeloptere Lompe.

Cyclopterus lumpus, Linnaeus.

A specimen from (iulf of St. Lawrence.

(B) Common Senlpin. Le (irand Chaboissenu à dix-huit épine de l'Ainérique din Nord.

Acanthocottus octodecinspinosus, Mitchill.

Two specimens from (inlf of $\mathrm{s}$. Lawrence.

(C) Sea Raven. L'Hémitriptère.

Hemitripterus americanus, Gmelin.

A specimen from Atlantic Coast of Canada.

943 Wolf-fish. L'Anarrhique ioup.

Auarrhichas lupus, Idinnaeus.

A specimen from (iulf of St. Lawrence.

944 Burbot or Ling. Ial Lotte Commnne.

Lota maculosa, Le Suenr.

Four specimens from Rock Lake, Haliburton Connty, Ontario.

From Mr. Andrew Fleck, of Parry Sound Rnilway.

945 Cusk. Le Brosme.

Brosmius brosinc, Muller.

Two specimens from Atlantic coast of Canada.

946 (A) Haddock. I'Fglefin.

Melanogrammus aglifinus, Linneus.

(13) White-hake or Codling.. La Merluche Blanche.

Physis tenuis, Mitchilu.

Specimen of each from Halifax, Nova Scotia. 
9.7 (A) Tom-eod or Font-fish. La l'otite Morme.

Mirrogudus tomeot, Walbmum.

Four specimens from Inalibx, Novil Scotia

(B) Cod-lish. Ia Morme.

Gadns callarins, Linmens.

A specimen from tinlf of st. Lawrence.

948 Cod-fish. La Morue.

Gadus callurias, Limmuss.

A specinen from Halifax, Nova feotia.

949 Halibut. Le Flétan on Halibnt.

Hippogtossus hippoglossus, liunirus.

Specimen froul fult of St. Lawreuce.

950 Rough Dab. Ia Limande.

Hippoglossoides platessoides, Fabricins.

Two specimens from ciulf of st. Lawrence.

951 (A) Black Sole. Ia Sole Noir.

Lepidopsetta bitineatu, ayres.

(B) California Flounder. Le: Flet de Catifornie.

Platichthys steltatus, Pallas.

A specimen of each from coast of liritish Colımbia.

American Lobster. Le Homard d'Ameri,fue.

Homarus americallis, Fiwards.

952 Male from (irand Manau, New Brmowick.

Loaned by Mr. Heury (iatehouse, of Montreal.

953 Two specimens from Lagoon at (irand Entry, Magdalen Islands, Province of Quebec. (( ollected by Commander Wakeham),

A specimen from (iabarns, Cape Breton. (From Mr. II. E.
Baker).

954 Specimen from Arichat, ('ape Breton. (Through Commander
Spain).

Crabs. Lees Crabes.

Chionocctes opilio, Kroyer.

955 Two specimeus from mouth of Gaspá Bay, Province of Quebec. 
Chionoccles opilio, Kroyer.

956 (A), (D) 'Two specimens from month of (iaspé Bay, Province of Quebec.

(B) Cancer borealis, Stimpson.

A specimen from Port Daniel, Bay Chaleur. " La Canadienne

(C) Lithodes maia, Latr.

A specimen from Westport, Nova scotia.

957 Chionocates opilio, Kroyer.

958 Cancer magister, Dana.

A specimen from Victoria, Vancouver Island.

MARINE INVERTEBRATES, SMALL FISHES, ETC. ARRANGED IN CASES IN A SERIES OF TRAYY.

Tray 1.

CASE A.

Sponge. Eponge.

959 Suberites compacta, Verrill.

A specimen from Sable Island, Nova Scotia.

\section{CGLENTERATES. ACALEPHES.}

960 Alcyonium rubiforme, Ehrenberg.

A number of specimens from Orphan Bank, Gulf of St. Lawrence. "La Canadienne" dredgings, 1999.

961 Spiny Sea-feather. Pennatulc.

Pennatula aculeata, Daniellsen

Two Specimens from Gulf of St. Lawrence.

POLYZOANS. BRYOZOAIRES.

Tray 2.

Myriozoum subgracile, d'Orbigny.

962 Fragments from Giulf of St. Lawrence.

963 Fragınents from Nouvelle, Bay Chaleur. " "La Canadienne

964 Flustra, sp.

A specimen from Rimonski, Province of Quebec. “La Cana-
dienne dreclgings, 1899.", 
Tray 3.

965 Cellepora corvicornis.

Fragments from Orphan Bank, Gulf of St. Lawrence.

966 Cellepora incrassata.

A spocimen from Orphan Bank, Gulf of St. Lawrence.

967 Eschara elegantula, d'Orbigny. A specimen on a Pecten shell, and fragments from Orphan Bank,
Gulf of St. Lawrence.

\section{ASCIDIANS OR TUNICATES. TUNIGIFRs.}

968 Cynthia pyriformis, Rathke.

A specimen from Metis, Province of Quebec.

Pelonaia arenifera, Stimpson.

969 Two specimens fron Richibucto, Straits of Northumberland.

970 Boltenia clavata.

A specimen from Metis, Province of Quebec.

t. Law-

\section{BRACHIOPODS OR LAMP SHELLS. BRACHIOPODES}

Tray 4.

971 Terebratulina septentrionalis, Gray.

A specimen from Bay of Fundy.

972 Terebratella spitzbergensis, Davidson.

A specimen and two valves, from Murray Bay, Province of

adienne

Rhynchonella psittacea, Gmelin.

973 A specimen with barnacles attached. "Hudson Bay Expedi-

974 Several specimens 'froin Cape "Gaspé Head. "La Canadienne
- dredgings, 1899." 


\section{OPHIURIANS. OPHIURIDES.}

Ophioglypha robusta, Lyman.

975 A number of specimens from Gulf of St. Lawrence.

Tray 5.

976 Two specimens from Rimouski, Province of Quebec. “ La Canadienne dredgings, 1899."

Ophioglypha sarsii, Lyman.

977 A specimen, and two dises, from near Churchill. "Hudson Bay Expedition, 1897.'

978 Ophiocoma aculeata, Muller. A specimen from near Churchill. "Hudson Bay Expedition,

979 Three specimens from Cape Gaspé Head. "La Canadienne dredgings, 1899."

980 Ophiocoma, sp.

A specimen from near Churchill. "Hudson Bay Expedition. 1897."

\section{STAR FISHES. ASTERIDES.}

Tray 6.

Solaster endeca, Forbes.

981 A specimen from Murray Bay, Province of Quebec.

982 A specimen from Cape (ìaspé Head, Province of Quebec. "La Canadienne dredgings, 1899."

983 Solaster papposus, Linniteus.

A specimen from Hudson Strait. "Hudson Bay Expedition, 1897."

Tray 7.

Asterias vulgaris, Stimpson .

984 Three specimens from Douglastown Head, one being 4-rayed. 985 Eight specimens from Paspebiac, Bay Chaleur, six being very
small.

Trays 8 and 9.

986 Five specimens from Pleasant Bay, Magdalen Islands.

"La Canadienue dredgings, 1899." 
Tray 10.

9872 specimens from Gaspé, Province of

Tray 11.

Asterias polaris, Muller and Troschel.

988 A specimen from Cape Gaspé Head, Province of Quebec.

Tray 12.

989 Two specimens from dienne dredgings, 1899." Province of Quebec. "La Cana-

Tray 13.

990 A specimen from Ungava Bay, "Hudson Bay Expedition, 1897.

\section{HOLOTHURIANS OR HOLOTHURIDES.}

Tray 14.

Sea Cucumbers.

Psolus phantapus, nken.

xpedition.

ec. " $\mathrm{La}$

xpedition, dredgings, 1899."

Trays 14 and 15.

Psolus squamatus.

992 Four specimens from Rimouski, Province of Quebec. "La Canadienne Dredgings, 18(9).",

SEA URCHINS AND SAND DOLLARS. ECHINIDES.

Tray 16.

Echinarachnius parma, Lamarck.

993 A number of specimens, denuded of spines, from Gulf of St Lawrence.

CASE B.

Tray 17.

rayed.

994 A number of specimens from Paspebiac, Bay Chaleur.

Trays 18, 19, 20, 21.

995 A number of specimens from Douglastown Ilead, Province of

Quebec : four being denuded of spines.

Tray 22.

996 A number of specimens fromt Pleasant Bay, Magdalen Islands.

"I a Canadienne dredgings, 1899,", 
Tray 23.

Strongylocentrotus drobachiensis, Müller.

997 Specimens among algae, etc., from Bird Rocks, Magdalen Islands.

- Tray 24.

998 Numerous specimens from Cape Gaspé Head.

Tray 25.

999. Five specimens from Rimouski, Province of Quebec.

Trays 26, 27, 28, 29.

1000 Thirteen specimens from Paspebiac, three being denuded of spines. "La Canadienne dredgings, 1899."

Tray 30.

1001 Six specimens, denuded of spines, from Bird Rocks, Magdalen Islands.

Tray 31 .

1002 Three specimens from Ungava Bay, "Hudson Bay Expedition 1897." One denuded of spines.

Tray 32.

11003 A specimen denuded of spines, from Grand Manan, New Brunswick. From Lt.-Col. Gourdeau, Deputy Minister of Marine and Fisheries.

Loxechinus purpuratus, Stimpson.

1004 Two specimens from near Victoria, Vancouver Island, one denuded of spines.

CASE C.

\section{LAMELLIBRANCHS. LAMELLIBRANCHES.}

Trays 33, 34, 35, 36 .

Hinnites giganteus, Gray.

1005 Two specimens (or four valves) from near Victoria, Vancouver Island.

Tray 37.

Pacific Oyster. Huitre de Pacific.

Ostrea lurida, Carpenter.

1006 A number of specimens from British Columbia. 
Tray 38.

1007 American Oyster. Huitre de Virginie.

Ostrea virginiana borealis, Lamarck.

A specimen (or two valves) from Prince Edward Island. From Mr. R. N. Venning, of Department of Marine and Fisheries.

Tray 39.

1008 Pecten caumnus, Giould.

A specimen (or two valves) with barnacles attached from Strait of Georgia, British Columbia.

Tray 40.

Pecten Islandicus, Linnæus.

1009 Three specimens (or five valves) from Northern part of Gulf of St. Lawrence.

Tray 41.

1010 Two specimens (or four valves) from Cape Gaspé Head, Gulf of St. Lawrence.

Trays 42-43.

1011 Four specinens (or eight valves) from Mal Bav, Gulf of St. Lawrence.

Tray 43.

1012 One very small specimen (or two valves) from Cape Gaspé Head, Gulf of St. Lawrence.

"La Canadienne Dredgings, 1899."

1013 Two valves from Grand Manan, New Brunswick. From Lt.-Col. Gourdeau, Deputy Minister of Marine and Fisheries.

Trays 44-45.

Pecten tenuicostatus, Mighels.

1014 One specimen (or two valves) from Gaspé Bay, Province of Quebec.

Trays 46-47.

1015 One specimen (or two valves) from Douglastown Bank, Province of Quebec, "La Canadienne dredgings, 1899," 
CASE I).

Modiola plicatula, Lamarck.

Tray 48.

1016 A number of specimens from Charlottetown, Prince Edward

Island. " La Canadienne dredgings, 1899."

Tray 49.

1017 One specimen (or two valves) from Pictou, Nova Scotia.

Modiola modiolus, Linnæus. 1018 Two specimens (or three valves) from Dougiastown Bank,
Province of Quebec.

Tray 50.

1019 A few valves from Pictou, Nova Scotia. "Ia Canadienne dredgings, i899."

Tray 51.

1020 One specimen (or two valves) from Nova Scotia.

Tray 52.

Mussel. Moule Comestible.

Mytilus edulis, Linnæus.
1021 One specimen (or two valves) from Metis, Province of Quebec.

1022 A few specimens from Charlottetown, Prince Edward Island. "La Canadienne dredgings, 1899.",

1023 A valve with barnacles attached, from Ungava Bay, "Hudson Bay Expedition, 1897."

Tray 53.

1024 A few valves from Port Daniel, Bay Chaleur, "La Canadienne dredgings, 1899."

Tray 54.

Mytilus californianus, Conrad.

1025 Two specimens (or four valves) from Victoria, Vancouver Island.

Tray 55.

Modiolaria corrugata, Stimpson.

1026 Three valves from Cape Gaspé Head, Gulf of St. Lawrence.

" La Canadienne dredgings," 1899."

Yoldia thraciaeformis.

1027 Two specimens from Gulf of St. Lawrence. 
Cardium islandicum, Linnseus.

1028 A specimen from Nouvelle, Bay Chaleur.

1029 Three specimens from Cape Gaspé Head, Gulf of St. Lawrence.

"La Canadienne dredgings, 1899."

Tray 56.

Serripes groenlandicus, Gmelin.

1030 Specimen of valve from Metis, Province of Quebec.

1031 Specimen of valve from Paroquet, Gulf of St. Lawrence.

1032 Specimen of valve from Orphan Bank, Magdalen Islands. "La Canadienne dredgings, 1899."

Cyprina islandica, Linnæus.

1033 A specimen (or two valves) from Bay of Fundy.

Tray 57.

Astarte lactea, Broderip and Sowerby.

1034 A specimen (or two valves) "Hudson Bay Expedition, 1897."

1035 A few specimens from Orphan Bank, Magdalen Islands. "La Canadienne dredgings, 1899."

Astarte banksii, Leach.

1036 Numerous specimens from Cape Gaspé Head, Gulf of St. Lawrence. "La Canadienne dredgings, 1899."

Astarte elliptica, Brown.

1037 A few specimens írom Orphan Bank, Magdalen Islands. "La Canadienne dredgings, 1899."

Callista convexa, Say.

1038 A specimen (or two valves) from Pleasant Bay, Magdalen Islands. "La Canadienne dredgings, 1899."

Tray 58.

Mercenaria violacea, Schumacher.

1039 Three specimens (or four valves) from Pictoú, Nova Scotia.

Tray 59.

Mactra ovalis, Gould.

1040 Two specimens (or three valves) from Gaspé, Province of Quebec. 
Tray 60.

Mactra solidissima, Dillwyn.

1041 Two specimens (or three valves) from Bay of Fundy.

1042 Two small specimens from Pictou, Nova Scotia. “I Ia Canadienne dredgings, 1899."

Tray 61 .

Ceronia jauressii, Joannis.

1043 A few specimens from Metis, Province of Quebec.

Macoma calcarea, Chemitz.

1044 A few specimens from Orphan Bank, Magdalen Islands. “ La Canadienne dredgings, 1899."

Tray 62.

CASE E.

Razor Shell. Solenide.

Ensatella americana, Gould.

Tray 63.

Siliqua costata, Say.

1045 Two specimens (or four valves) from Metis, Province of Quebec.

1046 Two specimens from Bay of Fundy.

Cyrtodaria siliqua, Spengler.

1047 Four valves from Gulf of St. Lawrence.

Tray 64 .

Mya arenaria, Linnæus.

1048 Two specimens (or four valves) from Port Daniel, Bay Chaleur.

"La Canadienne dredgings, 1899."

1049 Numerous tiny specimens from Murray Harbour, Prince Edward Island. Collected by Mr. Ernest Kemp.

Tray 65.

Saxicava rugosa, Lamarck.

1050 A few specimens from Ungava Bay, "Hudson Bay Expedition 1897."

Zirphaea crispata, Linnæus.

1051 Specimen of valve, from Sable Island, Nova Scotia.

Triy 65.

1052 Specimen (or two valves) from Victoria, Vancouver Island. 


\section{GASTROPODS. GASTROPODES.}

Tray 66.

1053 Chiton emersonii, Couthouy.

A specimen from Riviere du Loup, Province of Quebec

1054 Katherina tunicata, Gray.

A specimen froin Victoria, Vancouver Island.

1055 Tonicella marmorea, Fabricius.

Three specimens from Ungava Bay. "Hudson Bay Expedition, 1897."

1056 Planorbis parvus, Say.

One specimen from Ottawa, Ontario. (Fresh water).

1057 Planorbis campanulatus, Say.

A number of specimens from Ottawa, Ontario. (Fresh water).

Limpet. Patelle.

1058 Acmaea testudinalis, Müller.

Six specimens from Tadousac, Province of Quebec.

'Tray 67.

1059 A number of specimens from Grand Manan, New Brunswick. From Lt.-Col. Gourdeau, Deputy Minister of Marine and Fisheries.

1060 Haliotis Kamtschatkana, Jonas.

Two specinnens from Queen Charlotte Islands, British Columbia.

Tray 68.

Margarita cinerea, Couthuoy.

1061 A few specimens from Ungava Bay, "Hudson Bay Expedition, 1897."

1062 A few specinens from Cape Gaspé Head. "La Canadienue dredgings, 1849."

1063 Crepidula fornicata, Lamarck.

A number of specimens from Pictou, Nova Scotia.

Tray 69.

1064 Pachypoma gibberosum, Chemnitz.

I wo specimens from Victoria, Vancouver Island. 
1065 Lunatia lezvisii, Gould.

A specimen from Victoria, Vanconver Island.

Lunatia heros, Say.

1066 A specimen from Grand Mnıin, New Brunswick. From Lt.-Col . Gourdean, Depnty Minister of Marine and Fisheries.

Tray 70.

1067 Three specimens, and an operculum, froun Pictou, Nova Scotia.

Tray 71. 1068 Three small specim ins from Paspebiac, Bay Chaleur. " La
Canadienne dredgings, 1899."

Periwinkle. Littorine Littorale.

1069 Littorina littorea, Linnæus.

Four Specimens from Grand Manan, New Brunswick. From

Lt.-Col. Gonrdean, Deputy Minister of Marine and Fisheries.

1070 A few specimens from Georgetown, Prince Edward Island.

1071 A few specimens from Picton, Nova Scotia. "La Canadienne dredgings, 1899."

1072 Littorina rudis, Maton. Numerous specimens from Pictou, Nova Scotia. "La Cana-
dienne dredgings, 1899."

Tray 72.

Pelican's Foot. Pied de Pelican.

Aporrhais occidentalis, Beck. 1073 Four specimens from Ungava Bay. "Hudson Bay Expedition, 1074 A few specimens from Nouvelle, Bay Chaleur. "La Cana-
dienne dredgings, 1899."

Tray 73.

Dog Whelk. Pourpre a Teintnre.

Purpura lipillus, Lamarck.

1075 A few specimens from Metis, Province of Quebec:

1076 Two specimens, with opercula, from Columbine Bank, Magdalen Islands. "La Canadienne dredgings, 1899."

$10: 7$ Cerostoma foliatum, Gmelin.

T'wo specimens from Queen Charlotte Islands,!Britislı Columbia. 
Tray 74.

1078 Tritia obsoleta, Say. Four specimens from Pictou, Nova Scotia. "Ia Canadienne
dredgings, 1899."

1079 Buccinum tenue, Gray.

Two specimens from Metis, Province of Quebec.

1080 Buccinum plectrum, Stimpson.

Twe specimens from Metis, Provincé of Quebec.

Whelk. Buccin Onde.

1081 Buccinum undatum, Linnaeus.

Twu specimens from Metis, Province of Quebec.

Tray 75.

1052 Priene oregonensis, Redfield.

A specinen from Victoria, British Columbia.

1093 Sipho kroyeri, Muller.

Three specimens from Metis, Province of Quebec.

1084 Neptunea decemcostata, Say.

Two specimens from Rimouski, Province of Quebec; one with hermit-crab.

"La Canadienne dredgings, 1899."

Tray 76.

1085 Fusus dec ncostalus, Say.

One specimen from Grand Manan, New Brunswick. From Lt.Col. Gourdeau, Deputy Minister of Marine and Fisheries.

1086 Spindle-sliell.

Fusus islandicus, Gould.

One specimen from Grand Manan, Now Brunswick. From Lt.Col. Gourdeau, Deputy Minister of Marine and Fisheries.

1087 Tropi n scalariforme, Gould.

Four specine from Metis, Province of Quebec.

CASE F.

CRUSTACEANS. LES CRUSTACEANS.

Trays 77, 78, 79.

1088 Cancer borealis, Stimpson.

Three specime 11 , and a few specimens of carapaces from Port Daniel, Bay Chaleur. 
Trays 80 and 81 .

Hyas aranea, Linuaeus.

1089 Numerous specimens of various sizes from Paroquet, Province

'Trny 82.

1090 One small specimen from Orphan Bank, Magdalen Islands.

"Ia Canademe dredgings, 1899."

1091 Two specinens and carapace with barnacles attaclıed. "Hudson Bay Experlition, 1897."

Trays 83, 84, 85 .

Chionocactes opillio, Kroyer.

1092 Five specimens from Orphan Bank, Magdalen Islands. "Ia Canadienne Dredgings, 1899."

Tray 86.

Panopeus, sp.

1093 Two specimens from Uchuelet, Vancouver Island.

Epialtus productus, Randall.

1094 Three specimens from Victoria, Vanconver Island.

Tray 87.

Hermit Crab,

Eupagurus bernhardus.

1095 Three specimens, two being in shells of Gastroporls from Ungava Bay. " Hudson Bay Expedition, 1897."

Arcturus baffinii, Sabine.

1096 A specimen from near Churchill; " Iudson Bay Expedition

Salve Bug.

Aga psora, Kroyer.

1097 Three specimens from Churchill. "Hudson Bay Expedition,

1098. One specimen from Grand Manan, New Brunswick. From Lt.Col. Gourdeau, Deputy Minister of Marine and Fisheries.

1099 Two specimens from Canadian Fishery Museum.

Tray 88.

Coronula diadema, Blainville.

1100 Five specimens from the back of a whale, being still attached 
Numerous speoimens of Barnacles (Balanus) attached to shells of bivalve-mollusks and to stongs.

Tray 89.

1101 Sinall specimens on valve of Maclra solidissima, from Pictou, Nova Scotia.

1102 Three specimens from a large stone from Pasbebiac, Bay Chaleur.

1103 Two elongated specimens, united, froin Gulf of St. Lawrence.

Tray 90.

1101 A number of specimens on stones and on valve of Mactra ovalis, from Paroquet, Province of Quebec. "La Canadionnc dredginge, 1899."

Tray 9i.

1105 A number of specimens from Alberni Canal, Vancouver Island.

Lepas fascicularis, Ellis and Solander.

1106 A few specimens on alga from the Pacific Coast.

Tray 91.

FISHIES.

1107 Otoliths of God-fish.

Gadus callarias, Linnaeus..

Specimens from Port Daniel, Bay Chaleur.

Otolitlis of Fresh Water Drum.

Aplodinotus grunniens, Rafinesque.

1108 Two specimens froln Detroit River, Ontario.

1109 Two specimens from Ottawa River.

Tray 92.

Aspidophoroides monopterygius, Bloch.

1110 Two specimens from the Gulf of St.Lawrence. "La Canadienne dredgings, 1899."

Acanthocottus octodecimspinosus, Mitchill.

1111 A specimen from Cape Egmont, Straits of Northumberland. " La Canadieune dredgings, 1899."

Zoarces anguillaris, Peck.

1112 A specimen from Gaspé Bay, Province of Quebec. "La Canadienne dredgings, 1899."

Hippocampus hudsonius, DeKay .

1113 A specimen from Atlantic Coast of Canada. 
OYSTERS FROM PRINCE EDWARD ISLAND

Collected by Mr. Ernest Kemp, Canadian

American Oyster. Huitre de Virginie.

Oyster Expert.

Ostrea virginiana borealis, Lamarck.

CASE G.

Trays 93-96.

1114 A number of small specimens from Curtain Island, Richmond
Bay.

Trays 97-98.

1115 Two specimens or four valves, from Migell River.

Tray 99.

1116 Five specimens from Orwell Bay.

Tray 100.

1117 One specimen, or two valves, from Pinette River.

Trays 101-103.

1118 Three specimens, or six valves, from Bedeque Bay.

Trays 104-107.

1119 Eight specimens from Bedeque Bay.

Trays 108-122.

CASE H.

1120 Eight specimens, or sixteen valves, from Bedeque Bay.

MISCELLANAOUS SPECIMENS.

Shells of Lamellibranch's, collected by Mr. Ernest Kemp, Canadian Oyster Expert.

1121 American Oyster. Huitre de Virginie.

Ostrea virginiana borealis, Lamarck.
Specimeny from Migell and Morell Rivers, Prince Edward Island

11:2 Quahog or Hard Clam. Le Quahog.

Mercenaria violacea, Schumacher.

Specimens from Richmond Bay, Prince Edward Island. 
1123 Soft Clam. Mye des Sables.

Mya arenaria, Linnieus.

Specimens from Murray River, Prince Edward Island.

1124 Giant Clam or Sea Clam. Moule de Mer.

Mactra solidissima, Chemnitz.

Specimens from Murray Harbonr, Prince Ed ward Island.

11.25.Mussel.. Moule Comestible.

Mytilus edulis, Linnreus.

Two specimens, or four valves, from Richmond Bay, Prince Edward Island.

1126 Specimens of Valves of Pecten islandicus, L., one valve with egg-capsules of Buccinum undatum, L., attached, from Anticosti Island, Province of Quebec.

1127 Crytochiton stelleri, Middendorf.

A specimen from Victoria, Vancouver Island.

1128 Basket-fish. L'Astrophyte.

Astrophyton agassizii, Stimpson.

A specimen from Gulf of St. Lawrence.

1129 Star-fish. L'Astérie.

Asterias polaris, Müller and Troschel.

A specimen from Nouvelle, Bay Chaleur.

A specimen from Mal Bay, Province of Qnebec.

"La Canadienne dredgings, 1899."

A specimen from Gaspé.

1130 Sand-dollar. Echinarachnie Parına.

Echinarachnius parma, Lamarck.

A series of specimens from Douglastown Bank, Pro ince of Quebec.

" La Canadienne dredgings, 1899."

1131 Sea-urchin. Toxopneuste.

Loxechinus purpuratus, Stimpson.

Two specimens from Victoria, British Colmmbia, one being denuded of spines.

1132 Sponge. Eponge.

Halichondria.

A specimen from Grand Manan, New Brunswick. From Lt. -Col. Gourdeau, Deputy Minister of Marinc and Fisheries. 
MODELS OF CANOES AND FISHING GEAR.

1133 Two models of Canoes, Ontario Canoe Co., Peterboro, Ontario. 1134 Two Indian Paddles,

1135 Model of Cedar Canoe,

113 Model of Cedar Canoe, with 3 paddles, from British Columbia.

1137. Model of Scow from Yarmouth, Nova Scotia.

1138 Model of Ice-boat from Prince Edward Island.

1139 Ten Indian spears.

1140 Two shields with Fishing Gear.

924 Henri Menier, No. 8, Rue Alfred de Vigny, Paris, France.

1141 A Collection of Deep Sea Fishes from Anticosti Island, Province of Quebec.

925 Luc Dupues, Les Aulnaies, Que.

,Fish Oil. Huile de Poisson. 
rio. 
